\title{
Improving the Asymptotic Properties of Discrete System Zeros in Fractional-Order Hold Case
}

\author{
Cheng Zeng, ${ }^{1,2}$ Shan Liang, ${ }^{1,3}$ and Yingying Su${ }^{1,4}$ \\ ${ }^{1}$ College of Automation, Chongqing University, Chongqing 400044, China \\ ${ }^{2}$ Department of Mathematics, Guiyang University, Guiyang 550005, China \\ ${ }^{3}$ Key Laboratory of Dependable Service Computing in Cyber Physical Society Ministry of Education, \\ Chongqing University, Chongqing 400044, China \\ ${ }^{4}$ Department of Electric and Electronic Information Engineering, Chongqing University of Science and Technology, \\ Chongqing 401331, China
}

Correspondence should be addressed to Shan Liang; lightsun@cqu.edu.cn

Received 17 July 2013; Revised 11 October 2013; Accepted 21 October 2013

Academic Editor: Baocang Ding

Copyright (C) 2013 Cheng Zeng et al. This is an open access article distributed under the Creative Commons Attribution License, which permits unrestricted use, distribution, and reproduction in any medium, provided the original work is properly cited.

Remarkable improvements in the asymptotic properties of discrete system zeros may be achieved by properly adjusted fractionalorder hold (FROH) circuit. This paper analyzes asymptotic properties of the limiting zeros, as the sampling period $T$ tends to zero, of the sampled-data models on the basis of the normal form representation of the continuous-time systems with FROH. Moreover, when the relative degree of the continuous-time system is equal to one or two, an approximate expression of the limiting zeros for the sampled-data system with FROH is also given as power series with respect to a sampling period up to the third-order term. And, further, the corresponding stability conditions of the sampling zeros are discussed for fast sampling rates. The ideas of the paper here provide a more accurate approximation for asymptotic zeros, and certain known achievements on asymptotic behavior of limiting zeros are shown to be particular cases of the results presented.

\section{Introduction}

Zeros, along with poles, are fundamental characteristics of linear time-invariant systems, and the stability of zeros is one of the most important issues in the model matching and adaptive control problems. When a continuous-time system is discretized by the use of a sampler and a hold, the mapping between the discrete-time poles and their continuous-time counterparts is very simple; namely, stability of poles is reserved. There is unfortunately no simple transformation between the discrete-time zeros and their continuous-time ones because the zeros of discrete-time systems depend on sampling period $T$ [1]. More precisely, it is generally impossible to tranform a continuous-time system with zeros in the left-half plane to a discrete-time system with zeros inside the unit circle. That is to say, the stability of zeros is not necessarily preserved except in special cases. Therefore, one of the special cases (i.e., the limiting case) is that the sampling period $T$ tends to zero which has attracted considerable attention from the engineering point of view.

Perhaps the first attempt to study discrete system zeros was given by Åström and coworkers [1], who describe the asymptotic behavior of the discrete-time zeros for fast sampling rate when the original continuous-time plant is discretized with zero-order hold ( $\mathrm{ZOH})$. In this case, the discretized zeros are further called limiting zeros which are composed of the intrinsic zeros and sampling zeros [2]. The former ones have counterparts in the underlying continuoustime system and go to unity [3] while the latter ones, which have no continuous-time counterparts and are generated in the sampling process, go toward roots of a certain polynomial $[4,5]$ determined by relative degree of the continuous-time system.

In much of discussion about the properties of discretetime zeros, $\mathrm{ZOH}$ has been mainly employed as a hold circuit since it is used most commonly in practice $[1,3,6-10]$. 
Taking into account the fact that the type of hold circuit used critically influences the position of zeros, it is an interesting problem to investigate the zeros in the case of various holds. Hagiwara et al. [4] have carried out a comparative study and demonstrated that a first-order hold $(\mathrm{FOH})$ provides no advantage over $\mathrm{ZOH}$ as far as the stability of zeros of the resulting discrete-time systems is concerned. Passino and Antsaklis [11] have considered the fractional-order hold (FROH) as an alternative to the $\mathrm{ZOH}$ and shown that it can locate the zeros of discrete-time system inside the unit circle by some examples while $\mathrm{ZOH}$ fails to do so. In the very motivating work by Ishitobi [12], the properties of limiting zeros with FROH have been analyzed, and the corresponding pulse-transfer function has been also derived.

Moreover, Ishitobi has definitely presented the relationship between the relative degree and discretized zeros behavior when the continuous-time systems have the relative degree up to five for sufficiently small sampling periods. Further, Bàrcena et al. $[13,14]$ and Liang et al. $[15,16]$ have extended Ishitobi's results [12] from different angles and methods by investigating the limiting zeros in the case of a FROH. In addition, the limiting FROH zeros [12] have been also extended by Blachuta [17], who describes the accuracy of the asymptotic results for both the intrinsic and sampling zeros in terms of Bernoulli numbers and parameters of the continuous-time transfer function for sufficiently small sampling periods $T$.

In FROH case, the intrinsic zeros are located inside (resp., outside) the unit circle for small sampling periods when the corresponding continuous-time zeros lie strictly in the lefthalf plane (resp., right). For sampling zeros, at least one of the zeros lies strictly outside the unit circle if the relative degree of a continuous-time transfer function is greater than or equal to three $[12,18]$. This fact indicates that even though all the zeros of such a continuous-time system are stable, the corresponding discrete-time system has at least one unstable zero in the limiting case as the sampling period tends to zero. Thus, attention is here focused on continuous-time systems with relative degree less than or equal to two. More specifically, the corresponding discrete-time plants have one or two sampling zero(s) in the case of a FROH when the relative degree of a continuous-time transfer function is one or two. However, in these cases, the sampling zeros are located just on the unit circle, that is, in the marginal case of the stability. More importantly, it is a valuable research topic to find the criteria which guarantee that stable discretized zeros are obtained. Thus, the asymptotic behavior of the sampling zeros is an interesting issue as we explore the stability properties of the sampling zeros by analyzing the asymptotic properties as the sampling period tends to zero.

The objective of this paper is to analyze the improved asymptotic properties of the limiting zeros for discrete-time models by using a new kind of method. More precisely, we give an approximate expression of limiting zeros for the sampled-data system on the basis of the normal form representation of continuous-time system with $\mathrm{FROH}$ as power series with respect to a sampling period up to the third-order term when the relative degree of the continuous-time system is one or two. Our results include also the finding of how close limiting zeros are to the actual intrinsic and sampling ones, irrespectively of whether they are stable or not. The approach used could be referred to as an extension of that of $[12,17$, 18 , and one of the principal contribution in this paper, in particular, would consequently propose an analytical method to obtain the FROH zeros as stable as possible, or with improved asymptotic properties even when unstable, for a given continuous-time plant. Finally, we further discuss the stability of the sampling zeros for sufficiently small sampling periods, and some interesting examples are given to validate the main results.

\section{Sampled-Data Models with FROH}

Consider an $n$th continuous-time system with relative degree one or two described by a transfer function

$$
G(s)=K \frac{N(s)}{D(s)}, \quad K \neq 0
$$

where

$$
\begin{array}{r}
N(s)=s^{m}+b_{m-1} s^{m-1}+b_{m-2} s^{m-2}+\cdots+b_{0}, \\
m=n-1 \text { or } n-2, \\
D(s)=s^{n}+a_{n-1} s^{n-1}+a_{n-2} s^{n-2}+\cdots+a_{0} .
\end{array}
$$

The paper treats systems with relative degree one or two because at least one of the limiting zeros is unstable when the relative degree is greater than or equal to three though it is slightly a limitation.

2.1. Case of Relative Degree One $(m=n-1)$. The normal form of (1) with the relative degree one, $m=n-1$ is represented with an input $u$ and an output $y[19,20]$ as

$$
\begin{gathered}
\dot{\xi}=-d \xi+K u-\omega, \\
\dot{\boldsymbol{\eta}}=P \boldsymbol{\eta}+\mathbf{q} \xi, \\
y=\xi
\end{gathered}
$$

where

$$
\begin{gathered}
\boldsymbol{\eta}=\left[\begin{array}{lll}
\boldsymbol{\eta}_{1} & \cdots & \boldsymbol{\eta}_{n-1}
\end{array}\right]^{T}, \\
\omega=\mathbf{c}^{T} \boldsymbol{\eta}, \quad \mathbf{c}=\left[\begin{array}{llll}
r_{0} & r_{1} & \cdots & r_{n-2}
\end{array}\right]^{T}, \\
P=\left[\begin{array}{cccc}
0 & 1 & & 0 \\
& & \ddots & \\
-b_{0} & \cdots & & -b_{n-2}
\end{array}\right], \quad \mathbf{q}=\left[\begin{array}{c}
0 \\
\vdots \\
0 \\
1
\end{array}\right],
\end{gathered}
$$

and the scalars $d$ and $r_{i}(i=0, \ldots, n-2)$ are obtained from

$$
\begin{gathered}
D(s)=Q(s) N(s)+R(s), \\
Q(s)=s+d, \\
R(s)=r_{n-2} s^{n-2}+r_{n-3} s^{n-3}+\cdots+r_{0},
\end{gathered}
$$




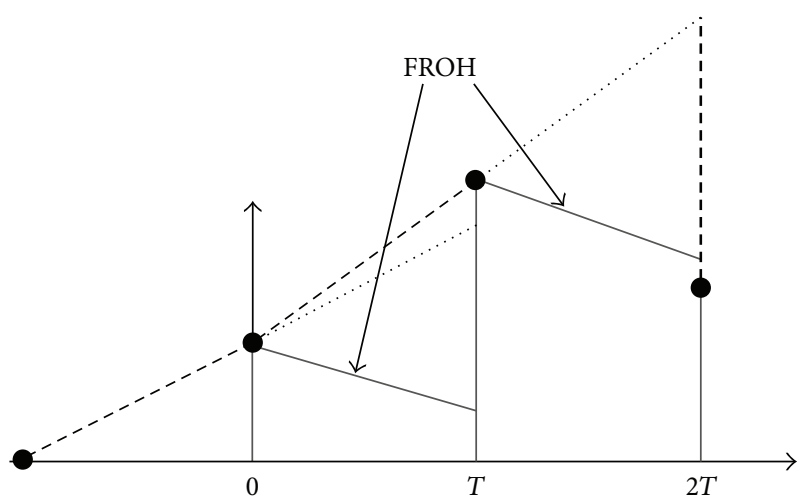

FIGURE 1: The signal reconstruction of a fractional-order hold with $\beta=-0.5$.

where

$$
\begin{gathered}
d=a_{n-1}-b_{n-2} \\
r_{i}=a_{i}-b_{i-1}-b_{i} d, \quad i=0, \ldots, n-2 .
\end{gathered}
$$

When the FROH signal reconstruction method is considered, the input is described by

$$
\begin{array}{r}
v(t)=u(k T)+\beta\left[\frac{u(k T)-u((k-1) T)}{T}\right](t-k T), \\
k T \leq t<(k+1) T, \quad k=0,1, \ldots,
\end{array}
$$

where $\beta$ is a real design parameter and $T$ is a sampling period $[11,12,18]$. It is obvious that $\mathrm{FROH}$ is reduced to $\mathrm{ZOH}$ for $\beta=0$ while it becomes the $\mathrm{FOH}$ for $\beta=1$. The signal reconstruction of a $\mathrm{FROH}$ with $\beta=-0.5$ is shown in Figure 1. have

Suppose $u(t)=v(t)$, and when a FROH is applied, we

$$
\dot{u}(t)=\beta\left[\frac{u(k T)-u((k-1) T)}{T}\right], \quad \ddot{u}(t)=\cdots=0 .
$$

Furthermore, (3) leads to the derivatives of the output

$$
\begin{gathered}
\dot{y}=-d \xi+K u-c^{T} \boldsymbol{\eta} \\
\ddot{y}=\left(d^{2}-\mathbf{c}^{T} \mathbf{q}\right) \xi-d K u+\left(d \mathbf{c}^{T}-\mathbf{c}^{T} P\right) \boldsymbol{\eta}+K \dot{u} \\
y^{(3)}=\left(-d^{3}+2 d \mathbf{c}^{T} \mathbf{q}-\mathbf{c}^{T} P \mathbf{q}\right) \xi+\left(d^{2}-\mathbf{c}^{T} \mathbf{q}\right) K u \\
+\left(d \mathbf{c}^{T} P-\mathbf{c}^{T} P^{2}-d^{2} \mathbf{c}^{T}+\mathbf{c}^{T} \mathbf{q} \mathbf{c}^{T}\right) \boldsymbol{\eta}-d K \dot{u} \\
y^{(4)}=\left\{d^{4}-3 d^{2} \mathbf{c}^{T} \mathbf{q}+2 d \mathbf{c}^{T} P \mathbf{q}-\mathbf{c}^{T} P^{2} \mathbf{q}+\left(\mathbf{c}^{T} \mathbf{q}\right)^{2}\right\} \xi \\
+\left(-d^{3}+2 d \mathbf{c}^{T} \mathbf{q}-\mathbf{c}^{T} P \mathbf{q}\right) K u \\
+\left\{d^{3} \mathbf{c}^{T}-2 d \mathbf{c}^{T} \mathbf{q} \mathbf{c}^{T}+\mathbf{c}^{T} P \mathbf{q} \mathbf{c}^{T}+d \mathbf{c}^{T} P^{2}\right. \\
\left.\quad-\mathbf{c}^{T} P^{3}-d^{2} \mathbf{c}^{T} P+\mathbf{c}^{T} \mathbf{q} \mathbf{c}^{T} P\right\} \boldsymbol{\eta}+\left(d^{2}-\mathbf{c}^{T} \mathbf{q}\right) K \dot{u}
\end{gathered}
$$

which are expressed by $\xi, \boldsymbol{\eta}$, and $K u$. Further, the derivatives of $\boldsymbol{\eta}$ are also represented by $\xi, \boldsymbol{\eta}$, and $K u$ as

$$
\begin{gathered}
\dot{\boldsymbol{\eta}}=P \boldsymbol{\eta}+\mathbf{q} \xi, \\
\ddot{\boldsymbol{\eta}}=(P \mathbf{q}-\mathbf{q} d) \xi+\left(P^{2}-\mathbf{q} \mathbf{c}^{T}\right) \boldsymbol{\eta}+\mathbf{q} K u, \\
\boldsymbol{\eta}^{(3)}=\left(P^{2} \mathbf{q}-\mathbf{q} \mathbf{c}^{T} \mathbf{q}-P \mathbf{q} d+\mathbf{q} d^{2}\right) \xi+(P \mathbf{q}-\mathbf{q} d) K u \\
+\left(P^{3}-\mathbf{q} \mathbf{c}^{T} P-P \mathbf{q} \mathbf{c}^{T}+\mathbf{q} \mathbf{c}^{T} d\right) \boldsymbol{\eta}+\mathbf{q} K \dot{u} .
\end{gathered}
$$

Hence, by substituting (9)-(15) into the right-hand side of

$$
y_{k+1}=\sum_{i=0}^{\infty} \frac{T^{i}}{i !} y_{k}^{(i)}
$$

$$
\boldsymbol{\eta}_{k+1}=\sum_{i=0}^{\infty} \frac{T^{i}}{i !} \boldsymbol{\eta}_{k}^{(i)}
$$

and defining the state variables $x_{k}=\left[y_{k}, \boldsymbol{\eta}_{k}^{T}\right]^{T}$, where the subscript $k$ denotes $t=k T$, the discrete-time state equations are definitely obtained. It is easy to show that zeros of a discrete-time system for a transfer function (1) are derived from (16).

Now, by applying the explicit expressions of $y_{k}, \dot{y}_{k}$, $\ldots, y_{k}^{(4)}$ and $\boldsymbol{\eta}_{k}, \ldots, \boldsymbol{\eta}_{k}^{(3)}$, the zeros of (16) are analyzed as follows:

$$
\begin{aligned}
& y_{k+1}=\sum_{i=0}^{4} \frac{T^{i}}{i !} y_{k}^{(i)}+O\left(T^{5}\right) \\
& =\left(1-d T+\frac{d^{2}-r_{n-2}}{2} T^{2}+\frac{2 d r_{n-2}-d^{3}-\mathbf{c}^{T} P \mathbf{q}}{6} T^{3}\right. \\
& \left.+\frac{d^{4}-3 d^{2} r_{n-2}+2 d \mathbf{c}^{T} P \mathbf{q} r_{n-2}^{2}-\mathbf{c}^{T} P^{2} \mathbf{q}^{4}}{24} T^{4}\right) y_{k} \\
& +\left\{\left(1+\frac{\beta}{2}\right) T-\left(\frac{d}{2}+\frac{d \beta}{6}\right) T^{2}\right. \\
& +\frac{4 d^{2}-4 r_{n-2}-r_{n-2} \beta+d^{2} \beta}{24} T^{3} \\
& \left.+\frac{2 d r_{n-2}-d^{3}-\mathbf{c}^{T} P \mathbf{q}^{4}}{24} T^{4}\right\} u_{k} \\
& +\left\{-\frac{\beta}{2} T+\frac{d \beta}{6} T^{2}-\frac{\left(d^{2}-r_{n-2}\right) \beta}{24} T^{3}\right\} K u_{k-1} \\
& +\left(-\mathbf{c}^{T} T+\frac{d \mathbf{c}^{T}-\mathbf{c}^{T} P}{2} T^{2}\right. \\
& +\frac{d \mathbf{c}^{T} P-\mathbf{c}^{T} P^{2}+\left(r_{n-2}-d^{2}\right) \mathbf{c}^{T}}{6} T^{3}
\end{aligned}
$$




$$
\begin{gathered}
+\left(\left(\left(d^{3}-2 d r_{n-2}\right) \mathbf{c}^{T}+\mathbf{c}^{T} P \mathbf{q} \mathbf{c}^{T}+d \mathbf{c}^{T} P^{2}\right.\right. \\
\left.-\mathbf{c}^{T} P^{3}-d^{2} \mathbf{c}^{T} P+r_{n-2} \mathbf{c}^{T} P\right) \\
\left.\left.\times(24)^{-1}\right) T^{4}\right) \boldsymbol{\eta}_{k}+O\left(T^{5}\right) \\
\boldsymbol{\eta}_{k+1}=\sum_{i=0}^{3} \frac{T^{i}}{i !} \boldsymbol{\eta}_{k}^{(i)}+O\left(T^{4}\right) \\
=\left(\mathbf{q} T+\frac{P \mathbf{q}-\mathbf{q} d}{2} T^{2}\right. \\
\left.+\frac{P^{2} \mathbf{q}-r_{n-2} \mathbf{q}+\mathbf{q} d^{2}-P \mathbf{q} d}{6} T^{3}\right) y_{k} \\
+\left\{\left(\frac{\mathbf{q}}{2}+\frac{\mathbf{q} \beta}{6}\right) T^{2} \frac{P \mathbf{q}-\mathbf{q} d}{6} T^{3}\right\} K u_{k}-\frac{\mathbf{q} \beta}{6} T^{2} K u_{k-1} \\
+\left(I+P T+\frac{P^{2}-\mathbf{q} \mathbf{c}^{T}}{2} T^{2}\right. \\
\left.+\frac{P^{3}-\mathbf{q} \mathbf{c}^{T} P-P \mathbf{q} \mathbf{c}^{T}+d \mathbf{q} \mathbf{c}^{T}}{6} T^{3}\right) \boldsymbol{\eta}_{k}+O\left(T^{4}\right) .
\end{gathered}
$$

The reason why the explicit expressions of $y_{k}, \dot{y}_{k}, \ldots$, $y_{k}^{(4)}$ and $\boldsymbol{\eta}_{k}, \ldots, \boldsymbol{\eta}_{k}^{(3)}$ are used is to obtain the approximate expansion of the limiting zeros for the discrete-time system with the order $T^{3}$.

2.2. Case of Relative Degree Two $(m=n-2)$. The normal form of (1) with the relative degree two, $m=n-2$ is represented $[19,20]$ as

$$
\begin{gathered}
\dot{\boldsymbol{\xi}}=\left[\begin{array}{ll}
0 & 1 \\
0 & 0
\end{array}\right] \boldsymbol{\xi}+\left[\begin{array}{l}
0 \\
1
\end{array}\right]\left(K u-\omega-d_{0} \xi_{1}-d_{1} \xi_{2}\right), \\
\dot{\boldsymbol{\eta}}=P \boldsymbol{\eta}+\mathbf{q} \xi_{1}, \\
y=\left[\begin{array}{ll}
1 & 0
\end{array}\right] \boldsymbol{\xi},
\end{gathered}
$$

where

$$
\begin{aligned}
& \boldsymbol{\xi}=\left[\begin{array}{ll}
\xi_{1} & \xi_{2}
\end{array}\right]^{T}, \quad \boldsymbol{\eta}=\left[\begin{array}{lll}
\boldsymbol{\eta}_{1} & \cdots & \boldsymbol{\eta}_{n-2}
\end{array}\right]^{T}, \\
& \omega=\mathbf{c}^{T} \boldsymbol{\eta}, \quad \mathbf{c}=\left[\begin{array}{llll}
\mathbf{c}_{0} & \mathbf{c}_{1} & \cdots & \mathbf{c}_{n-3}
\end{array}\right]^{T}, \\
& P=\left[\begin{array}{cccc}
0 & 1 & & O \\
& O & \ddots & 1 \\
-b_{0} & \cdots & -b_{n-4} & -b_{n-3}
\end{array}\right], \quad \mathbf{q}=\left[\begin{array}{c}
0 \\
\vdots \\
0 \\
1
\end{array}\right],
\end{aligned}
$$

and the scalars $d_{i}(i=0,1)$ and $\mathbf{c}_{i}(i=0, \ldots, n-3)$ are obtained from

$$
\begin{gathered}
D(s)=Q(s) N(s)+R(s), \\
Q(s)=s^{2}+d_{1} s+d_{0}, \\
R(s)=\mathbf{c}_{n-3} s^{n-3}+\cdots+\mathbf{c}_{0},
\end{gathered}
$$

where

$$
\begin{gathered}
d_{0}=a_{n-2}-b_{n-4}-b_{n-3} d_{1}, \\
d_{1}=a_{n-1}-b_{n-3}, \\
\mathbf{c}_{i}=a_{i}-b_{i-2}-b_{i-1} d_{1}-b_{i} d_{0}, \quad i=0, \ldots, n-3 .
\end{gathered}
$$

When a FROH is used, the normal form (19) yields the derivatives of the output

$$
\begin{gathered}
\ddot{y}=K u-d_{0} \xi_{1}-d_{1} \xi_{2}-\mathbf{c}^{T} \boldsymbol{\eta}, \\
y^{(3)}=\left(d_{0} d_{1}-\mathbf{c}^{T} \mathbf{q}\right) \xi_{1}+\left(d_{1}^{2}-d_{0}\right) \xi_{2}-d_{1} K u \\
+\left(d_{1} \mathbf{c}^{T}-\mathbf{c}^{T} P\right) \boldsymbol{\eta}+K \dot{u}, \\
y^{(4)}=\left(d_{0}^{2}-d_{0} d_{1}^{2}+d_{1} \mathbf{c}^{T} \mathbf{q}-\mathbf{c}^{T} P \mathbf{q}\right) \xi_{1} \\
+\left(-\mathbf{c}^{T} \mathbf{q}+2 d_{0} d_{1}-d_{1}^{3}\right) \xi_{2} \\
+\left(d_{1}^{2}-d_{0}\right) K u-d_{1} K \dot{\boldsymbol{u}} \\
+\left\{-\left(d_{1}^{2}-d_{0}\right) \mathbf{c}^{T}+d_{1} \mathbf{c}^{T} P-\mathbf{c}^{T} P^{2}\right\} \boldsymbol{\eta}, \\
y^{(5)}=\left(d_{0} d_{1}^{3}-d_{1}^{2} \mathbf{c}^{T} \mathbf{q}+2 \mathbf{c}^{T} \mathbf{q} d_{0}-2 d_{0}^{2} d_{1}\right. \\
\left.\quad-\mathbf{c}^{T} P^{2} \mathbf{q}+d_{1} \mathbf{c}^{T} P \mathbf{q}\right) \xi_{1} \\
+\left(d_{0}^{2}-3 d_{0} d_{1}^{2}+2 d_{1} \mathbf{c}^{T} \mathbf{q}\right. \\
\left.\quad-\mathbf{c}^{T} P \mathbf{q}+d_{1}^{4}\right) \xi_{2} \\
+\left(-d_{1}^{3}+2 d_{0} d_{1}-\mathbf{c}^{T} \mathbf{q}\right) K u \\
+\left\{\mathbf{c}^{T} \mathbf{q} \mathbf{c}^{T}-2 d_{0} d_{1} \mathbf{c}^{T}+d_{1}^{3} \mathbf{c}^{T}\right. \\
\quad-\left(d_{1}^{2}-d_{0}\right) \mathbf{c}^{T} P+d_{1} \mathbf{c}^{T} P^{2} \\
\left.\quad-\mathbf{c}^{T} P^{3}\right\} \boldsymbol{\eta}+\left(d_{1}^{2}-d_{0}\right) K \dot{u} .
\end{gathered}
$$

Further, the derivatives of $\boldsymbol{\eta}$ are also represented as

$$
\begin{gathered}
\dot{\boldsymbol{\eta}}=P \boldsymbol{\eta}+\mathbf{q} \xi_{1}, \\
\ddot{\boldsymbol{\eta}}=P \mathbf{q} \xi_{1}+\mathbf{q} \xi_{2}+P^{2} \boldsymbol{\eta}, \\
\boldsymbol{\eta}^{(3)}=\left(P^{2} \mathbf{q}-\mathbf{q} d_{0}\right) \xi_{1}+\left(P \mathbf{q}-\mathbf{q} d_{1}\right) \xi_{2}+\mathbf{q} K u \\
+\left(P^{3}-\mathbf{q c} c^{T}\right) \boldsymbol{\eta},
\end{gathered}
$$




$$
\begin{aligned}
\boldsymbol{\eta}^{(4)}= & \left(-P \mathbf{q} d_{0}+\mathbf{q} d_{0} d_{1}+P^{3} \mathbf{q}-\mathbf{q} \mathbf{c}^{T} \mathbf{q}\right) \xi_{1} \\
& +\left(-\mathbf{q} d_{0}+P^{2} \mathbf{q}-P \mathbf{q} d_{1}+\mathbf{q} d_{1}^{2}\right) \xi_{2} \\
& +\left(P \mathbf{q}-\mathbf{q} d_{1}\right) K u+\mathbf{q} K \dot{u} \\
& +\left(-P \mathbf{q} \mathbf{c}^{T}+\mathbf{q} d_{1} \mathbf{c}^{T}+P^{4}-\mathbf{q} \mathbf{c}^{T} P\right) \boldsymbol{\eta} .
\end{aligned}
$$

Hence, by substituting (23)-(30) into the right-hand side of (16) and

$$
\dot{y}_{k+1}=\sum_{i=0}^{\infty} \frac{T^{i}}{i !} y_{k}^{(i+1)}
$$

and defining the state variables $x_{k}=\left[y_{k}, \dot{y}_{k}, \boldsymbol{\eta}_{k}^{T}\right]^{T}$, the discrete-time state equations are obtained.

Now, by using the explicit expressions of $y_{k}, y_{k}^{\prime}, \ldots, y_{k}^{(5)}$ and $\boldsymbol{\eta}_{k}, \ldots, \boldsymbol{\eta}_{k}^{(4)}$, the zeros of the discrete-time system (16) and (31) are analyzed as follows:

$$
\begin{aligned}
& y_{k+1}=\sum_{i=0}^{5} \frac{T^{i}}{i !} y_{k}^{(i)}+O\left(T^{6}\right) \\
& =\left(1-\frac{d_{0}}{2} T^{2}+\frac{d_{0} d_{1}-\mathbf{c}^{T} \mathbf{q}^{3}}{6} T^{3}\right. \\
& +\frac{d_{0}^{2}-d_{0} d_{1}^{2}+d_{1} \mathbf{c}^{T} \mathbf{q}-\mathbf{c}^{T} P \mathbf{q}}{24} T^{4} \\
& -\left(\left(2 \mathbf{c}^{T} \mathbf{q} d_{0}-2 d_{0}^{2} d_{1}+d_{0} d_{1}^{3}-d_{1}^{2} \mathbf{c}^{T} \mathbf{q}\right.\right. \\
& \left.-d_{1} \mathbf{c}^{T} P \mathbf{q}+\mathbf{c}^{T} P^{2} \mathbf{q}\right) \\
& \left.\left.\times(120)^{-1}\right) T^{5}\right) y_{k} \\
& +\left(T-\frac{d_{1}}{2} T^{2}+\frac{d_{1}^{2}-d_{0}}{6} T^{3}+\frac{2 d_{0} d_{1}-d_{1}^{3}-\mathbf{c}^{T} \mathbf{q}}{24} T^{4}\right. \\
& \left.+\frac{d_{0}^{2}-3 d_{0} d_{1}^{2}+2 d_{1} \mathbf{c}^{T} \mathbf{q}-\mathbf{c}^{T} P \mathbf{q}+d_{1}^{4}}{120} T^{5}\right) \dot{y}_{k} \\
& +\left\{\left(\frac{1}{2}+\frac{\beta}{6}\right) T^{2}-\frac{4 d_{1}+d_{1} \beta}{24} T^{3}\right. \\
& +\frac{5 d_{1}^{2}-5 d_{0}+\left(d_{1}^{2}-d_{0}\right) \beta}{120} T^{4} \\
& \left.+\frac{2 d_{0} d_{1}-\mathbf{c}^{T} \mathbf{q}-d_{1}^{3}}{120} T^{5}\right\} K u_{k} \\
& +\left\{-\frac{\beta}{6} T^{2}+\frac{d_{1} \beta}{24} T^{3}-\frac{\left(d_{1}^{2}-d_{0}\right) \beta}{120} T^{4}\right\} K u_{k-1}
\end{aligned}
$$

$$
\begin{gathered}
+\left\{-\frac{\mathbf{c}^{T}}{2} T^{2}+\frac{d_{1} \mathbf{c}^{T}-\mathbf{c}^{T} P}{6} T^{3}\right. \\
+\frac{d_{1} \mathbf{c}^{T} P-\left(d_{1}^{2}-d_{0}\right) \mathbf{c}^{T}-\mathbf{c}^{T} P^{2}}{24} T^{4} \\
+\left(\left(\mathbf{c}^{T} \mathbf{q} \mathbf{c}^{T}-2 d_{0} d_{1} \mathbf{c}^{T}+d_{1}^{3} \mathbf{c}^{T} d_{1} \mathbf{c}^{T} P^{2}\right.\right. \\
\left.-\left(d_{1}^{2}-d_{0}\right) \mathbf{c}^{T} P-\mathbf{c}^{T} P^{3}\right) \\
\left.\left.\times(120)^{-1}\right) T^{5}\right\} \boldsymbol{\eta}_{k}+O\left(T^{6}\right),
\end{gathered}
$$

$$
\begin{aligned}
& \dot{y}_{k+1}=\sum_{i=0}^{4} \frac{T^{i}}{i !} y_{k}^{(i+1)}+O\left(T^{5}\right) \\
& =\left(-d_{0} T+\frac{d_{0} d_{1}-\mathbf{c}^{T} \mathbf{q}^{2}}{2} T^{2}\right. \\
& +\frac{d_{0}^{2}-d_{0} d_{1}^{2}+d_{1} \mathbf{c}^{T} \mathbf{q}-\mathbf{c}^{T} P \mathbf{q}}{6} T^{3} \\
& +\left(\left(\mathbf{c}^{T} P^{2} \mathbf{q}-d_{1} \mathbf{c}^{T} P \mathbf{q}-2 \mathbf{c}^{T} \mathbf{q} d_{0}\right.\right. \\
& \left.+2 d_{0}^{2} d_{1}-d_{0} d_{1}^{3}+d_{1}^{2} \mathbf{c}^{T} \mathbf{q}\right) \\
& \left.\left.\times(24)^{-1}\right) T^{4}\right) y_{k} \\
& +\left(1-d_{1} T+\frac{d_{1}^{2}-d_{0}}{2} T^{2}+\frac{2 d_{0} d_{1}-d_{1}^{3}-\mathbf{c}^{T} \mathbf{q}}{6} T^{3}\right. \\
& \left.+\frac{d_{0}^{2}-3 d_{0} d_{1}^{2}+2 d_{1} \mathbf{c}^{T} \mathbf{q}-\mathbf{c}^{T} P \mathbf{q}+d_{1}^{4}}{24} T^{4}\right) \dot{y}_{k} \\
& +\left\{\left(1+\frac{\beta}{2}\right) T-\frac{3 d_{1}+d_{1} \beta}{6} T^{2}\right. \\
& +\frac{4 d_{1}^{2}-4 d_{0}+\left(d_{1}^{2}-d_{0}\right) \beta}{24} T^{3} \\
& \left.+\frac{2 d_{0} d_{1}-\mathbf{c}^{T} \mathbf{q}-d_{1}^{3}}{24} T^{4}\right\} K u_{k} \\
& +\left\{-\frac{\beta}{2} T+\frac{d_{1} \beta}{6} T^{2}\right. \\
& \left.-\frac{\left(d_{1}^{2}-d_{0}\right) \beta}{24} T^{3}\right\} K u_{k-1} \\
& +\left\{-\mathbf{c}^{T} T+\frac{d_{1} \mathbf{c}^{T}-\mathbf{c}^{T} P}{2} T^{2}\right. \\
& +\frac{d_{1} \mathbf{c}^{T} P-\mathbf{c}^{T} P^{2}-\left(d_{1}^{2}-d_{0}\right) \mathbf{c}^{T}}{6} T^{3}
\end{aligned}
$$




$$
\begin{aligned}
& +\left(\left(\mathbf{c}^{T} \mathbf{q} \mathbf{c}^{T}-2 d_{0} d_{1} \mathbf{c}^{T}+d_{1}^{3} \mathbf{c}^{T}\right.\right. \\
& \left.-\left(d_{1}^{2}-d_{0}\right) c^{T} P-c^{T} P^{3}+d_{1} c^{T} P^{2}\right) \\
& \left.\left.\times(24)^{-1}\right) T^{4}\right\} \boldsymbol{\eta}_{k}+O\left(T^{5}\right) \text {, } \\
& \boldsymbol{\eta}_{k+1}=\sum_{i=0}^{4} \frac{T^{i}}{i !} \boldsymbol{\eta}_{k}^{(i)}+O\left(T^{5}\right) \\
& =\left(\mathbf{q} T+\frac{P \mathbf{q}^{2}}{2} T^{2}+\frac{P^{2} \mathbf{q}-\mathbf{q} d_{0}}{6} T^{3}\right. \\
& \left.+\frac{P^{3} \mathbf{q}-\mathbf{q} \mathbf{c}^{T} \mathbf{q}-P \mathbf{q} d_{0}+\mathbf{q} d_{0} d_{1}}{24} T^{4}\right) y_{k} \\
& +\left(\frac{\mathbf{q}}{2} T^{2}+\frac{P \mathbf{q}-\mathbf{q} d_{1}}{6} T^{3}+\frac{P^{2} \mathbf{q}-\mathbf{q} d_{0}}{24} T^{4}\right. \\
& \left.+\frac{-P \mathbf{q} d_{1}+\mathbf{q} d_{1}^{2}}{24} T^{4}\right) \dot{y}_{k}-\frac{\mathbf{q} \beta}{24} T^{3} K u_{k-1} \\
& +\left\{\left(\frac{\mathbf{q}}{6}+\frac{\mathbf{q} \beta}{24}\right) T^{3}+\frac{P \mathbf{q}-\mathbf{q} d_{1}}{24} T^{4}\right\} K u_{k} \\
& +\left(I+P T+\frac{P^{2}}{2} T^{2}+\frac{P^{3}-\mathbf{q c}^{T}}{6} T^{3}\right. \\
& \left.+\frac{P^{4}-P \mathbf{q} \mathbf{c}^{T}-\mathbf{q} \mathbf{c}^{T} P-P \mathbf{q c}^{T}}{24} T^{4}\right) \boldsymbol{\eta}_{k}+O\left(T^{5}\right) .
\end{aligned}
$$

$$
\begin{aligned}
& \left.-\frac{2 d r_{n-2}-d^{3}-\mathbf{c}^{T} P \mathbf{q}-5 \mathbf{c}^{T} P \mathbf{q} \beta+2 \mathbf{c}^{T} \mathbf{q} d \beta}{24} T^{3}\right] z \\
& +\frac{\beta}{2}-\frac{d \beta}{6} T+\frac{\left(d^{2}-r_{n-2}\right) \beta}{24} T^{2} \\
& \left.-\frac{5 \mathbf{c}^{T} P \mathbf{q} \beta-2 \mathbf{c}^{T} \mathbf{q} d \beta}{24} T^{3}\right\} \\
& \times\left|(1-z) I+P T+\frac{P^{2}}{2} T^{2}+\frac{2 P^{3}+\mathbf{q} \mathbf{c}^{T} P}{12} T^{3}\right|=0 .
\end{aligned}
$$

Proof. The limiting zeros of the discrete-time system (16) are equivalent to zeros in (17) and (18), which are given by substituting $y_{k}=y_{k+1}=0$ into (17) and (18) as follows:

$$
M_{1}\left[\begin{array}{c}
K U_{k-1} \\
H \\
K U_{k}
\end{array}\right]=\mathbf{0}_{n},
$$

where $U_{k-1}, H$, and $U_{k}$ are the $z$-transforms of $u_{k-1}, \boldsymbol{\eta}_{k}$, and $u_{k}$, respectively, and the matrix $M_{1}$ is defined by

$$
M_{1}=\left[\begin{array}{ccc}
m_{11} & \mathbf{m}_{12}^{T} & m_{13} \\
-z & \mathbf{0}^{T} & 1 \\
\mathbf{m}_{31} & M_{32} & \mathbf{m}_{33}
\end{array}\right],
$$

with

$$
\begin{aligned}
m_{11}= & T \bar{m}_{11}+O\left(T^{4}\right), \\
\mathbf{m}_{12}^{T}= & T \overline{\mathbf{m}}_{12}^{T}+O\left(T^{5}\right), \\
m_{13}= & T \bar{m}_{13}+O\left(T^{5}\right), \\
\bar{m}_{11}= & -\frac{\beta}{2}+\frac{d \beta}{6} T-\frac{\left(d^{2}-r_{n-2}\right) \beta}{24} T^{2}, \\
\overline{\mathbf{m}}_{12}^{T}= & -\mathbf{c}^{T}+\frac{d \mathbf{c}^{T}-\mathbf{c}^{T} P}{2} T+\frac{d \mathbf{c}^{T} P-\mathbf{c}^{T} P^{2}}{6} T^{2} \\
& +\frac{\left(r_{n-2}-d^{2}\right) \mathbf{c}^{T}}{6} T^{2}+\frac{\left(d^{3}-2 d r_{n-2}\right) \mathbf{c}^{T}}{24} T^{3} \\
& +\frac{\mathbf{c}^{T} P \mathbf{q} \mathbf{c}^{T}+d \mathbf{c}^{T} P^{2}-\mathbf{c}^{T} P^{3}}{24} T^{3} \\
& +\frac{-d^{2} \mathbf{c}^{T} P+r_{n-2} \mathbf{c}^{T} P}{24} T^{3}, \\
\bar{m}_{13}= & \left(1+\frac{\beta}{2}\right)-\left(\frac{d}{2}+\frac{d \beta}{6}\right) T \\
& +\left\{\frac{d^{2}-r_{n-2}}{6}+\frac{\left(d^{2}-r_{n-2}\right) \beta}{24}\right\} T^{2} \\
& +\frac{-d^{3}+2 d r_{n-2}-\mathbf{c}^{T} P \mathbf{q}_{1}}{24} T^{3},
\end{aligned}
$$




$$
\begin{aligned}
\mathbf{m}_{31}= & -\frac{\mathbf{q} \beta}{6} T^{2}+O\left(T^{4}\right), \\
M_{32}= & (-z+1) I+P T+\frac{P^{2}-\mathbf{q} \mathbf{c}^{T}}{2} T^{2} \\
& +\frac{d \mathbf{q} \mathbf{c}^{T}-P \mathbf{q} \mathbf{c}^{T}+P^{3}-\mathbf{q} \mathbf{c}^{T} P}{6} T^{3}+O\left(T^{4}\right), \\
\mathbf{m}_{33}= & \left(\frac{\mathbf{q}}{2}+\frac{\mathbf{q} \beta}{6}\right) T^{2}+\frac{P \mathbf{q}-\mathbf{q} d}{6} T^{3}+O\left(T^{4}\right) .
\end{aligned}
$$

Thus, the zeros are derived from

$$
\left|M_{1}\right|=0 \text {. }
$$

From the relationship

$$
M=M_{1}\left[\begin{array}{lll}
1 & 0 & 0 \\
0 & 1 & 0 \\
1 & 0 & 1
\end{array}\right],
$$

it is obvious that the condition $|M|=0$ is equivalent to $\left|M_{1}\right|=0$.

Expanding the result along the second row leads to the following equation:

$$
\begin{aligned}
|M| & =-(-z+1)\left|\begin{array}{ll}
\mathbf{m}_{12}^{T} & m_{13} \\
M_{32} & \mathbf{m}_{33}
\end{array}\right| \\
& -\left|\begin{array}{ll}
m_{11}+m_{13} & \mathbf{m}_{12}^{T} \\
\mathbf{m}_{31}+\mathbf{m}_{33} & M_{32}
\end{array}\right| \\
& =T\left[-z\left|A_{1}\right|+(z-1)\left|A_{2}\right|\right]=0,
\end{aligned}
$$

where

$$
\begin{aligned}
\left|A_{1}\right|= & {\left[1-\frac{d}{2} T+\frac{d^{2}-r_{n-2}}{6} T^{2}\right.} \\
& \left.+\frac{2 d r_{n-2}-d^{3}-\mathbf{c}^{T} p \mathbf{q}^{3}}{24} T^{3}\right] \\
& \times\left|(1-z) I+P T+\frac{P^{2}}{2} T^{2}+\frac{2 P^{3}+\mathbf{q c}^{T} P}{12} T^{3}\right| \\
= & \Delta_{1} \times \Delta_{2}, \\
\left|A_{2}\right|= & {\left[-\frac{\beta}{2}+\frac{d \beta}{6} T-\frac{\left(d^{2}-r_{n-2}\right) \beta}{24} T^{2}\right.} \\
& \left.+\left(\frac{5 \mathbf{c}^{T} p \mathbf{q} \beta-2 \mathbf{c}^{T} \mathbf{q} d \beta}{24}\right) T^{3}\right] \\
& \times\left|(1-z) I+P T+\frac{P^{2}}{2} T^{2}+\frac{2 P^{3}+\mathbf{q} \mathbf{c}^{T} P}{12} T^{3}\right| \\
= & \bar{\Delta}_{1} \times \Delta_{2} .
\end{aligned}
$$

Then,

$$
|M|=\left[\left(\bar{\Delta}_{1}-\Delta_{1}\right) z-\bar{\Delta}_{1}\right] \times \Delta_{2} .
$$

Hence, the approximate values of limiting zeros of the discrete-time system are obtained as the roots of (35).

Remark 2. Equation (35) implies that an approximation of the sampling zero is expressed as

$$
\begin{aligned}
& {\left[-1-\frac{\beta}{2}+\left(\frac{d}{2}+\frac{d \beta}{6}\right) T\right.} \\
& -\left(\frac{d^{2}-r_{n-2}}{6}+\frac{\left(d^{2}-r_{n-2}\right) \beta}{24}\right) T^{2} \\
& \left.+\frac{2 d r_{n-2}-d^{3}-\mathbf{c}^{T} P \mathbf{q}-5 \mathbf{c}^{T} P \mathbf{q} \beta+2 \mathbf{c}^{T} \mathbf{q} d \beta}{24} T^{3}\right] z \\
& +\frac{\beta}{2}-\frac{d \beta}{6} T+\frac{\left(d^{2}-r_{n-2}\right) \beta}{24} T^{2} \\
& -\frac{5 \mathbf{c}^{T} P \mathbf{q} \beta-2 \mathbf{c}^{T} \mathbf{q} d \beta}{24} T^{3}=0,
\end{aligned}
$$

and the approximate values of the intrinsic zeros are derived from

$$
\left|(1-z) I+P T+\frac{P^{2}}{2} T^{2}+\frac{2 P^{3}+\mathbf{q} \mathbf{c}^{T} P}{12} T^{3}\right|=0 .
$$

Remark 3. Theorem 1 is applicable to also the case of multiple zeros of the continuous-time system (1) with FROH and further gives approximate values with higher order of accuracy than those of the previous result [17].

Remark 4. An insightful observation in Theorem 1 is that it has a form of a correction to the asymptotic result of Ishitobi $[12,18]$ in the form of a power term of $T$. Similarly, the following result (Theorem 6) both the intrinsic zeros and sampling zeros is also clarified in a more precise manner than Ishitobi's result $[12,17,18]$ when the relative degree of continuous-time systems is two.

Remark 5. On the basis of the approach in [21], it is immediate to derive the asymptotic condition of the limiting zeros in the case of a FROH with relative degree one:

$$
\begin{aligned}
\left|M_{1}\right| \approx & \left\{\left(1+\frac{\beta}{2}\right)-\left(\frac{d}{2}+\frac{d \beta}{6}\right) T\right. \\
& +\frac{4 d^{2}-4 r_{n-2}+\left(d^{2}-r_{n-2}\right) \beta}{24} T^{2} \\
& \left.+\frac{2 d r_{n-2}-d^{3}-c^{T} P \mathbf{q}^{3}}{24} T^{3}\right\} \\
\times & {\left[-z+1-\frac{2}{2+\beta}+\frac{d \beta}{3(2+\beta)^{2}} T\right.} \\
& +\frac{\beta\left(6 r_{n-2}+3 r_{n-2} \beta-\beta d^{2}\right)}{18(2+\beta)^{3}} T^{2}
\end{aligned}
$$




$$
\begin{gathered}
+\left(\left(\beta \left(1014 d \beta r_{n-2}-348 d^{3}\right.\right.\right. \\
\left.+348 d r_{n-2}\right) \\
+\beta\left(1188 d \beta^{2} r_{n-2}-1026 d^{3} \beta\right. \\
\left.-1193 d^{3} \beta^{2}\right) \\
+\beta\left(36 \mathbf{c}^{T} P \mathbf{q} \beta+36 \mathbf{c}^{T} P \mathbf{q}\right. \\
\left.\left.+9 \mathbf{c}^{T} P \mathbf{q} \beta^{2}\right)\right) \\
\left.\left.\times\left(108(2+\beta)^{4}\right)^{-1}\right) T^{3}\right] \\
\times\left|(1-z) I+P T+\frac{P^{2}}{2} T^{2}+\frac{2 P^{3}+\mathbf{q} \mathbf{c}^{T} P}{12} T^{3}\right| .
\end{gathered}
$$

When the relative degree of continuous-time systems is one and the continuous-time input is generated by a FROH, further research is needed to establish connections between (46) and (35) of Theorem 1 in this paper, wherein the idea (35) has more decent effect than the literature [21] in terms of techniques in studying the discrete system zeros.

3.2. Case of Relative Degree Two $(m=n-2)$. Next, we present asymptotic properties of limiting zeros of discrete-time control system in the case of a FROH as power series with respect to a sampling period up to the third-order term when the relative degree of the continuous-time system is two. An approximate expression, in fact, of zeros of a discrete-time system is derived from (32)-(34), and the other results of this paper are given by the following Theorem.

Theorem 6. The zeros of a discrete-time system for the continuous-time transfer function (1) with FROH are given for $T \ll 1$ approximately by the roots of

$$
\begin{gathered}
\left\{\left[\frac{1}{2}+\frac{\beta}{6}-\left(\frac{4 d_{1}+d_{1} \beta}{24}\right) T+\frac{5 d_{1}^{2}-5 d_{0}}{120} T^{2}\right.\right. \\
\left.+\frac{\beta d_{1}^{2}-\beta d_{0}}{120} T^{2}+\frac{2 d_{0} d_{1}-\mathbf{c}_{n-3}-d_{1}^{3}}{120} T^{3}\right] z^{2} \\
+\left[\frac{3+\beta}{6}-\frac{2 d_{1}+d_{1} \beta}{6} T\right. \\
+\frac{15 d_{1}^{2}-5 d_{0}+8 \beta d_{1}^{2}-3 \beta d_{0}}{120} T^{2} \\
+\left(\frac{d_{0} d_{1}}{40}+\frac{\mathbf{c}_{n-3}}{120}-\frac{d_{1}^{3}}{30}+\frac{d_{0} d_{1} \beta}{180}\right. \\
\left.\left.+\frac{\mathbf{c}_{n-3} \beta}{72}-\frac{d_{1}^{3} \beta}{80}\right) T^{3}\right] z
\end{gathered}
$$

$$
\begin{aligned}
& -\frac{\beta}{3}+\frac{5 d_{1} \beta}{24} T+\frac{4 d_{0} \beta-9 d_{1}^{2} \beta}{120} T^{2} \\
& \left.+\frac{9 d_{1}^{3}-4 d_{0} d_{1} \beta-10 \mathbf{c}_{n-3} \beta}{720} T^{3}\right\} \\
& \times\left|(1-z) I+P T+\frac{P^{2}}{2} T^{2}+\frac{P^{3}}{6} T^{3}\right|=0 .
\end{aligned}
$$

Proof. Zeros of the discrete-time system (16) and (31), equivalent to (32)-(34), are given by substituting $y_{k}=y_{k+1}=0$ into (32)-(34) as follows:

$$
M_{2}\left[\begin{array}{c}
Y_{d} \\
K U_{k-1} \\
H \\
K U_{k}
\end{array}\right]=\mathbf{0}_{n}
$$

where $Y_{d}$ is the $z$-transforms of $\dot{y}_{k}$ and the matrix $M_{2}$ is defined by

$$
M_{2}=\left[\begin{array}{cccc}
m_{11} & m_{12} & \mathbf{m}_{13}^{T} & m_{14} \\
m_{21} & m_{22} & \mathbf{m}_{23}^{T} & m_{24} \\
0 & -z & \mathbf{0}^{T} & 1 \\
\mathbf{m}_{41} & \mathbf{m}_{42} & M_{43} & \mathbf{m}_{44}
\end{array}\right]
$$

with

$$
\begin{aligned}
& m_{11}=T \bar{m}_{11}+O\left(T^{5}\right) \text {, } \\
& m_{12}=T \bar{m}_{12}+O\left(T^{5}\right) \text {, } \\
& \mathbf{m}_{13}^{T}=T \overline{\mathbf{m}}_{13}^{T}+O\left(T^{5}\right), \\
& m_{14}=T \bar{m}_{14}+O\left(T^{5}\right) \text {, } \\
& \bar{m}_{11}=1-\frac{d_{1}}{2} T+\frac{d_{1}^{2}-d_{0}}{6} T^{2} \\
& +\frac{-d_{1}^{3}+2 d_{0} d_{1}-\mathbf{c}_{n-3}}{24} T^{3}, \\
& \bar{m}_{12}=-\frac{\beta}{6} T+\frac{d_{1} \beta}{24} T^{2}-\frac{\left(d_{1}^{2}-d_{0}\right) \beta}{120} T^{3} \\
& \overline{\mathbf{m}}_{13}^{T}=-\frac{\mathbf{c}^{T}}{2} T+\frac{d_{1} \mathbf{c}^{T}-\mathbf{c}^{T} P}{6} T^{2} \\
& +\frac{-\left(d_{1}^{2}-d_{0}\right) \mathbf{c}^{T}+d_{1} c^{T} P-c^{T} P^{2}}{24} T^{3}, \\
& \bar{m}_{14}=\left(\frac{1}{2}+\frac{\beta}{6}\right) T+\left(-\frac{d_{1}}{6}-\frac{d_{1} \beta}{24}\right) T^{2} \\
& +\left[\frac{d_{1}^{2}-d_{0}}{24}+\frac{\left(d_{1}^{2}-d_{0}\right) \beta}{120}\right] T^{3},
\end{aligned}
$$




$$
\begin{aligned}
& m_{21}=-z+1-d_{1} T+\frac{d_{1}^{2}-d_{0}}{2} T^{2} \\
& +\frac{-d_{1}^{3}+2 d_{0} d_{1}-\mathbf{c}_{n-3}}{6} T^{3}+O\left(T^{4}\right) \\
& m_{22}=-\frac{\beta}{2} T+\frac{d_{1} \beta}{6} T^{2}-\frac{\left(d_{1}^{2}-d_{0}\right) \beta}{24} T^{3}+O\left(T^{4}\right), \\
& \mathbf{m}_{23}^{T}=-\mathbf{c}^{T} T+\frac{d_{1} \mathbf{c}^{T}-\mathbf{c}^{T} P}{2} T^{2} \\
& +\frac{d_{1} \mathbf{c}^{T} P-\left(d_{1}^{2}-d_{0}\right) \mathbf{c}^{T}-\mathbf{c}^{T} P^{2}}{6} T^{3}+O\left(T^{4}\right), \\
& m_{24}=\left(1+\frac{\beta}{2}\right) T+\left(-\frac{d_{1}}{2}-\frac{d_{1} \beta}{6}\right) T^{2} \\
& +\left(\frac{d_{1}^{2}-d_{0}}{6}+\frac{\left(d_{1}^{2}-d_{0}\right) \beta}{24}\right) T^{3}+O\left(T^{4}\right), \\
& \mathbf{m}_{41}=\frac{\mathbf{q}}{2} T^{2}+\frac{P \mathbf{q}-\mathbf{q} d_{1}}{6} T^{3}+O\left(T^{4}\right), \\
& \mathbf{m}_{42}=-\frac{\mathbf{q} \beta}{24} T^{3}+O\left(T^{4}\right), \\
& M_{43}=(-z+1) I+P T+\frac{P^{2}}{2} T^{2}+\frac{P^{3}-\mathbf{q c}^{T}}{6} T^{3} \\
& +O\left(T^{4}\right) \\
& \mathbf{m}_{44}=\left(\frac{\mathbf{q}}{6}+\frac{\mathbf{q} \beta}{24}\right) T^{3}+O\left(T^{4}\right) .
\end{aligned}
$$

Thus, the zeros are derived from

$$
\left|M_{2}\right|=0
$$

From the relationship

$$
\bar{M}=M_{2}\left[\begin{array}{llll}
1 & 0 & 0 & 0 \\
0 & 1 & 0 & 0 \\
0 & 0 & 1 & 0 \\
0 & 1 & 0 & 1
\end{array}\right],
$$

it is obvious that the condition $|\bar{M}|=0$ is equivalent to $\left|M_{2}\right|=0$.

Expanding the result along the third row leads to the following equation:

$$
\begin{aligned}
|\bar{M}| & =-(-z+1)\left|\begin{array}{lll}
m_{11} & \mathbf{m}_{13}^{T} & m_{14} \\
m_{21} & \mathbf{m}_{23}^{T} & m_{24} \\
\mathbf{m}_{41} & M_{43} & \mathbf{m}_{44}
\end{array}\right| \\
& -\left|\begin{array}{lll}
m_{11} & m_{12}+m_{14} & \mathbf{m}_{13}^{T} \\
m_{21} & m_{22}+m_{24} & \mathbf{m}_{23}^{T} \\
\mathbf{m}_{41} & \mathbf{m}_{42}+\mathbf{m}_{44} & M_{43}
\end{array}\right| \\
& =T\left[-z\left|\bar{A}_{1}\right|+(-z+1)\left|\bar{A}_{2}\right|\right]=0
\end{aligned}
$$

where

$$
\begin{aligned}
\left|\bar{A}_{1}\right|= & {\left[\frac{1}{2}-\frac{d_{1}}{6} T+\frac{d_{1}^{2}-d_{0}}{24} T^{2}\right.} \\
& \left.+\frac{-\mathbf{c}_{n-3}+2 d_{0} d_{1}-d_{1}^{3}}{120} T^{3}\right] \\
& \times \\
& \left.+\frac{d_{1}^{3}+3 d_{0} d_{1}-9 \mathbf{c}_{n-3}}{270} T^{3}\right] \\
\times & \left|(1-z) I+P T+\frac{P^{2}}{2} T^{2}+\frac{P^{3}}{6} T^{3}\right| \\
= & \Delta_{1} \times \Delta_{2} \times \Delta_{3}, \\
\left|\bar{A}_{2}\right|= & \left(\frac{\beta}{6} T-\frac{d_{1} \beta}{24} T^{2}+\frac{\left(d_{1}^{2}-d_{0}\right) \beta}{120} T^{3}\right) \\
\times & \left.+\left(\frac{7 d_{0} d_{1}}{240}-\frac{3 d_{1}^{3}}{960}-\frac{\mathbf{c}_{n-3}}{12}\right) T^{3}\right] \\
\times & {\left[-z-2+\frac{3 d_{1}}{4} T+\left(-\frac{13 d_{1}^{2}}{80}+\frac{d_{0}}{10}\right) T^{2}\right.} \\
& {\left[\begin{array}{l}
P_{2}^{2} \\
2
\end{array} T^{2}+\frac{P^{3}}{6} T^{3} \mid\right.}
\end{aligned}
$$

Equations (54) and (55) will be calculated in the appendix. Then,

$$
|M|=\left[\left(-\bar{\Delta}_{1} \times \bar{\Delta}_{2}-\Delta_{1} \times \Delta_{2}\right) z+\bar{\Delta}_{1} \times \bar{\Delta}_{2}\right] \times \Delta_{3} .
$$

Hence, the approximate values of the zeros of the discretetime system are obtained as the roots of (47).

Remark 7. Equation (47) implies that the approximations of the sampling zeros are expressed as

$$
\begin{aligned}
& {\left[\frac{1}{2}+\frac{\beta}{6}-\left(\frac{d_{1}}{6}+\frac{d_{1} \beta}{24}\right) T+\frac{5 d_{1}^{2}-5 d_{0}+\beta d_{1}^{2}-\beta d_{0}}{120} T^{2}\right.} \\
& \left.+\frac{2 d_{0} d_{1}-\mathbf{c}_{n-3}-d_{1}^{3}}{120} T^{3}\right] z^{2} \\
& +\left[\frac{3+\beta}{6}-\frac{2 d_{1}+d_{1} \beta}{6} T\right. \\
& +\frac{15 d_{1}^{2}-5 d_{0}+8 \beta d_{1}^{2}-3 \beta d_{0}}{120} T^{2}
\end{aligned}
$$




$$
\begin{gathered}
+\left(\frac{d_{0} d_{1}}{40}+\frac{\mathbf{c}_{n-3}}{120}-\frac{d_{1}^{3}}{30}+\frac{d_{0} d_{1} \beta}{180}\right. \\
\left.\left.+\frac{\mathbf{c}_{n-3} \beta}{72}-\frac{d_{1}^{3} \beta}{80}\right) T^{3}\right] z \\
-\frac{\beta}{3}+\frac{5 d_{1} \beta}{24} T+\frac{4 d_{0} \beta-9 d_{1}^{2} \beta}{120} T^{2} \\
+\frac{9 d_{1}^{3}-4 d_{0} d_{1} \beta-10 \mathbf{c}_{n-3} \beta}{720} T^{3}=0
\end{gathered}
$$

and the approximate values of the intrinsic zeros are derived from

$$
\left|(1-z) I+P T+\frac{P^{2}}{2} T^{2}+\frac{P^{3}}{6} T^{3}\right|=0
$$

Remark 8. When FROH is implemented in practice, an approximate fractional-order hold (AFROH) using $\mathrm{ZOH}$ would be convenient practical solution. The basic idea of AFROH is that, at each sampling interval, the output of $\mathrm{FROH}$ is approximated by staircase waveforms that can be generated by $\mathrm{ZOH}[15,22]$ (see Figure 2). Therefore, an asymptotic expression of the limiting zeros in AFROH case is derived similarly.

In the particular case when the sampling period tends to zero, it is immediate to obtain the following Corollary although a similar result is also obtained by Ishitobi $[12,18]$.

\section{Corollary 9. One has the following cases.}

Case a. Assume that the relative degree of a continuous-time system is one. If $-1<\beta$ (resp., $\beta<-1$ ), then the sampling zero of the sampled-data model is stable (resp., unstable) in the case of a FROH when the sampling period tends to zero.

Case $b$. Assume that the relative degree of a continuous-time system is two. If $-1<\beta<0$ (resp., $\beta \leq-1$ or $\beta>0$ ), then the sampling zeros of the sampled-data model are stable (resp., unstable) in the case of a FROH when the sampling period tends to zero.

Proof. One has the following cases.

Case a. For $n-m=1$, we have from (35)

$$
A_{1}(z ; \beta)=\left(-1-\frac{\beta}{2}\right) z+\frac{\beta}{2} \text {. }
$$

Simple straightforward calculation will verify that the root of $A_{1}(z ; \beta)=0$ is stable if $-1<\beta$ and is unstable if $\beta \leq$ -1 . In addition, when $\beta=-1$, there remains the possibility

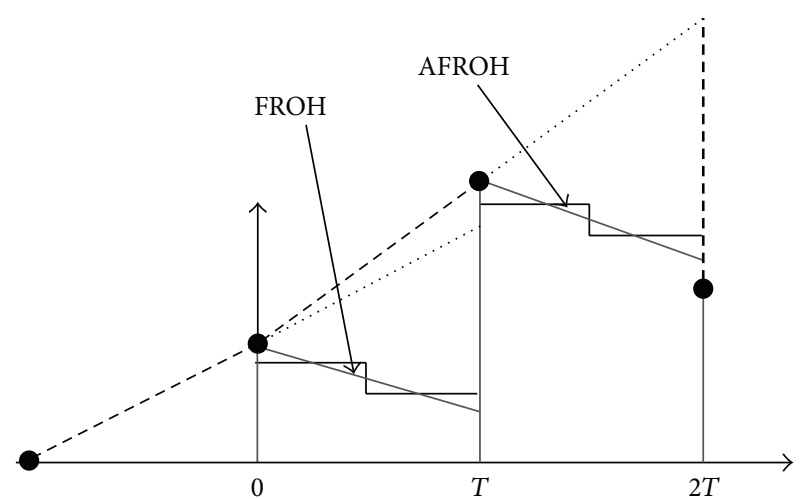

FIGURE 2: Output of the approximate fractional-order hold with $N=$ 2 and $\beta=-0.5$.

that the sampling zero corresponding to $A_{1}(z ; \beta)$, which approaches $z=-1$, lies inside the open unit disc as $T \rightarrow 0$.

Case $b$. For $n-m=2$, the polynomial $A_{2}(z ; \beta)$ is represented from (47) as

$$
A_{2}(z ; \beta)=\left(\frac{1}{2}+\frac{\beta}{6}\right) z^{2}+\left(\frac{1}{2}+\frac{\beta}{6}\right) z-\frac{\beta}{3} .
$$

When we perform the bilinear transformation $z=(\omega+$ $1) /(\omega-1)$ on the above equation, the polynomial is written as

$$
A_{2}(z ; \beta)=3 \omega^{2}+3(1+\beta) \omega-\beta=0 .
$$

It is clear that the two roots of (61) lie in the open left half of $\omega$-plane if $-1<\beta<0$, and at least one of them stays in the closed right-half plane if $\beta \leq-1$ or $\beta \geq 0$. In particular, only one of the sampling zeros approaches -1 at $\beta=0$. Namely, the stability of the sampling zeros is marginal, that is, in the case of a $\mathrm{ZOH}[4,23]$.

Remark 10. When the FROH signal reconstruction device is used, the parameter $\beta$ which is the device adjustable gain (generalised gain) is the major factor that decides the stability properties of sampling zeros of sampled-data systems with FROH. In other words, the appropriate $\beta$ is determined to obtain the FROH that provides sampling zeros as stable as possible, or with improved stability properties even when being unstable, for a given continuous-time plant.

Remark 11. If the relative degree of a continuous-time transfer function is two and the sum of the zeros is less than or equal to the sum of the poles, the limiting zeros of the sampled system with FROH of $-1<\beta<0$ stay definitely inside the unit circle while those with $\mathrm{ZOH}$ may lie outside or on the unit circle. Therefore, the FROH with $-1<\beta<0$ will produce all stable sampling zeros for a wider class of continuous-time plants than that of the $\mathrm{ZOH}$.

\section{Simulation Examples}

This section presents three interesting examples to show the stability of sampling zeros with FROH by improved 
TABLE 1: Intrinsic zeros of the sampled-data system with relative degree one.

\begin{tabular}{lcc}
\hline$T$ & Approximate values (35) & Exact values \\
\hline \multirow{2}{*}{0.01} & 0.980311498, & 0.980310411, \\
& 0.980082836 & 0.980084008 \\
0.02 & 0.961230229, & 0.961222048, \\
& 0.960324437 & 0.960333962 \\
0.05 & 0.907398436, & 0.907292742, \\
& 0.901893230 & 0.902049060 \\
0.1 & 0.827729968, & 0.827130532, \\
& 0.806603365 & 0.807949540 \\
0.2 & 0.697266611, & 0.695230664, \\
& 0.617400056 & 0.629936753 \\
\hline
\end{tabular}

TABLE 2: Sampling zero of the sampled-data system with relative degree one and $\beta=-1 / 2$.

\begin{tabular}{lcc}
\hline$T$ & Approximate values (35) & Exact values \\
\hline 0.01 & 0.329701686 & 0.329657909 \\
0.02 & 0.326139088 & 0.325821314 \\
0.05 & 0.316359822 & 0.314371422 \\
0.1 & 0.302545156 & 0.294211129 \\
0.2 & 0.283625731 & 0.248072696 \\
\hline
\end{tabular}

TABLE 3: Sampling zero of the sampled-data system with relative degree one and $\beta=1$.

\begin{tabular}{lcc}
\hline$T$ & Approximate values (35) & Exact values \\
\hline 0.01 & -0.331507563 & -0.331319933 \\
0.02 & -0.329739729 & -0.329258964 \\
0.05 & -0.324624858 & -0.322146285 \\
0.1 & -0.317193888 & -0.307047943 \\
0.2 & -0.306446658 & -0.255131263 \\
\hline
\end{tabular}

asymptotic properties. It has also shown that the stability of zeros will be improved by using FROH instead of $\mathrm{ZOH}$. Both kinds of zeros are calculated by applying MATLAB, and in the simulation figures (Figures 3, 4, 5, 6, 7, 8, and 9), the solid line and dotted line indicate the exact values and approximate values, respectively.

Example 1. Consider the following transfer function with the relative degree one [21]:

$$
G(s)=\frac{(s+2)^{2}}{s(s+1)(s-2)} .
$$

The approximate values (35) and the exact values of zeros of the sampled-data system for the transfer function (62) are shown in Tables 1-4 and corresponding figures, where the intrinsic zeros are shown in Table 1 and the sampling zero is respectively shown in Tables 2,3 , and 5 owing to the difference of the parameter $\beta$. Equation (35) gives good approximation also for the case of a continuous-time transfer function with $\mathrm{FROH}$.
TABLE 4: Sampling zero of the sampled-data system with relative degree one.

\begin{tabular}{lcc}
\hline$T$ & $\begin{array}{c}\text { Approximate values (46) } \\
\beta=-1 / 2\end{array}$ & $\begin{array}{c}\text { Approximate values (46) } \\
\beta=1\end{array}$ \\
\hline 0.01 & 0.3297 & -0.3315 \\
0.02 & 0.3262 & -0.3297 \\
0.05 & 0.3164 & -0.3246 \\
0.1 & 0.3025 & -0.3169 \\
0.2 & 0.2841 & -0.3046 \\
\hline
\end{tabular}

TABLE 5: Sampling zero of the sampled-data system with relative degree one and $\beta=-2$.

\begin{tabular}{lcc}
\hline$T$ & Approximate values (35) & Exact values \\
\hline 0.01 & 121.047619 & 120.640978703 \\
0.02 & 60.80588235 & 60.6072444662 \\
0.05 & 24.85125858 & 24.530135132 \\
0.1 & 13.10222222 & 12.387624318 \\
0.2 & 7.603157322 & 6.1116305864 \\
\hline
\end{tabular}

When the continuous-time systems have relative degree one, a discrete-time system corresponding to a continuoustime transfer function (62) has two intrinsic zeros and one sampling zero in the case of a FROH. In particular, the values of the intrinsic zeros with $\mathrm{FROH}$ are approximately equal to those with $\mathrm{ZOH}$ owing to the parameter $\beta$ (see also Remark 13). Further, the stability of sampling zero with FROH depends on the parameter $\beta$. When $-1<\beta$ (resp., $\beta<-1$ ), the sampling zero of the sampled-data model is stable (resp., unstable) in the case of a FROH for small sampling periods (see Tables 2-4).

Case $a(\beta=-1 / 2)$. See Table 2 and Figure 5 .

Case $b(\beta=1)$. See Table 3 and Figure 6 .

Case $c(\beta=-2)$. See Table 5. From the foregoing analysis, it is obvious that the limiting zeros of the sampled-data system with $\mathrm{FROH}$ of $-1<\beta$ are located inside the unit circle. In addition, (46) gives good approximation and the sampling zero lies inside the unit circle for small sampling periods at $\beta=-1 / 2$ and $\beta=1$ (see Table 4). Furthermore, it can be seen from the corresponding Tables $2-4$ that (35) can offer a more accurate approximation than that of (46) in terms of the stable sampling zero of discrete-time model.

Example 2. Consider a transfer function with the relative degree two

$$
G(s)=\frac{s+7}{(s+1)(s+2)(s+3)} .
$$

On the basis of the results in $[21,24]$, the stability condition of sampling zeros with $\mathrm{ZOH}$ is dissatisfied since $d_{1}=$ $a_{2}-b_{0}=-1<0$. However, the stability of the sampling zeros will be preserved in the case of a FROH when $-1<\beta<0$. The approximate values (47) and the exact values of zeros of the sampled-data system for the transfer function (63) are 


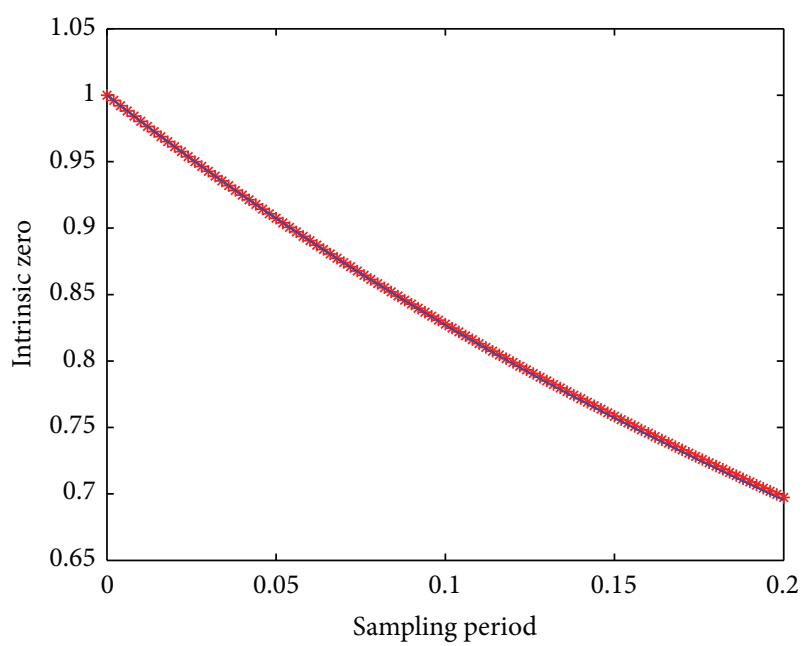

FIGURE 3: Intrinsic zero of sampled-data model with relative degree one.

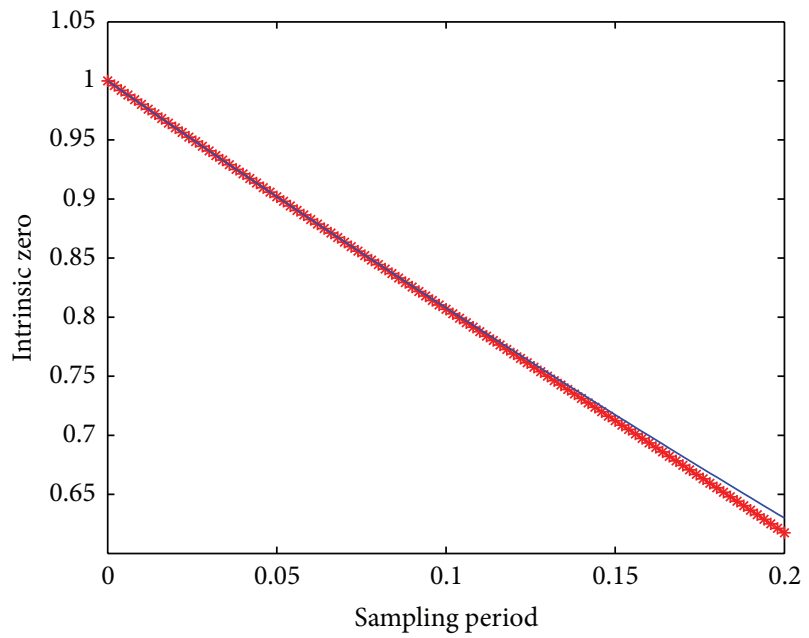

FIGURE 4: Intrinsic zero of sampled-data model with relative degree one.

TABLE 6: Intrinsic zero of the sampled-data system with relative degree two.

\begin{tabular}{lcc}
\hline$T$ & Approximate values (47) & Exact values \\
\hline 0.01 & 0.9324 & 0.932347819 \\
0.02 & 0.8693 & 0.869400472 \\
0.05 & 0.7041 & 0.704589951 \\
0.1 & 0.4878 & 0.496541723 \\
0.2 & 0.1227 & 0.244801539 \\
\hline
\end{tabular}

shown in Tables 6-9, where the intrinsic zero is shown in Table 6 and the sampling zeros are shown in Tables 7, 8, and 9. Equation (47) gives good approximation and the sampling zeros lie inside the unit circle for small sampling periods with FROH, while $\mathrm{ZOH}$ fails to do so.

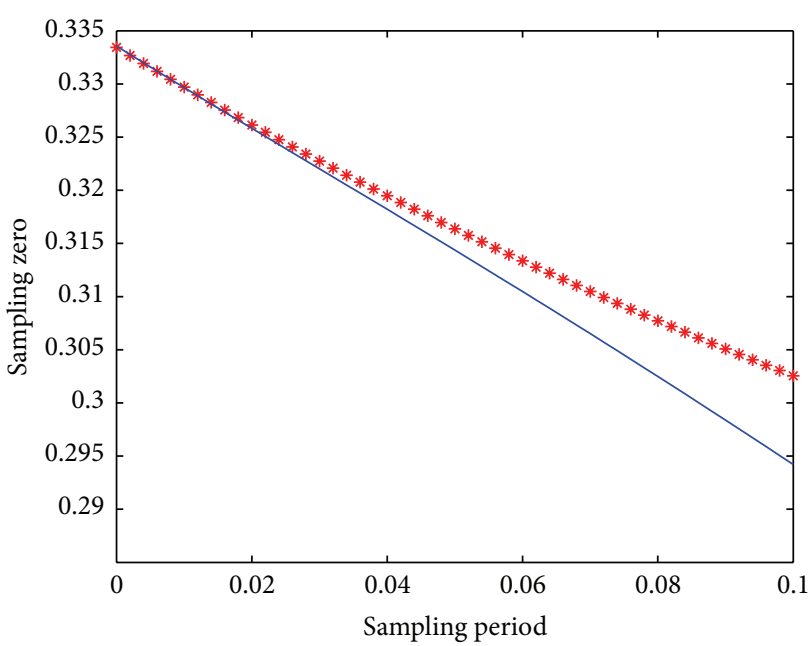

FIgURE 5: Sampling zero of sampled-data model with relative degree one and $\beta=-1 / 2$.

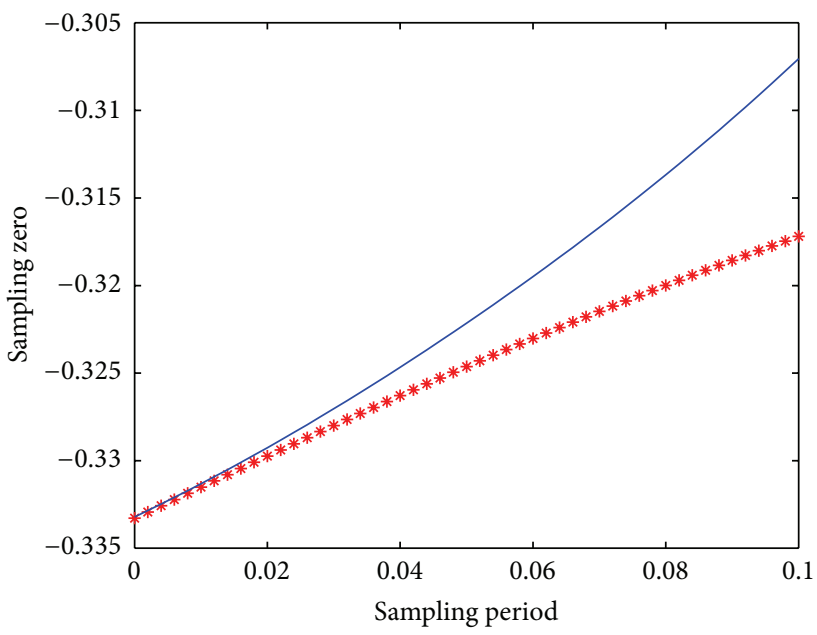

FIGURE 6: Sampling zero of sampled-data model with relative degree one and $\beta=1$.

Remark 12. From Examples 1 and 2, it can be obviously seen that $\mathrm{FROH}$ is reduced to $\mathrm{FOH}$ for $\beta=1$. The limiting zeros for sufficiently small $T$ in the case of a FOH are stable with relative degree one while it is unstable with relative degree two. Thus, a FOH provides no advantage over $\mathrm{ZOH}$ and FROH with the stability of the limiting zeros [4].

Remark 13. When the FROH signal reconstruction device is used, the parameter $\beta$, so called the device adjustable gain (generalised gain), is also a factor which affects the intrinsic zeros of sampled-data systems by numerically verifying in the case of a FROH. More precisely, it only affects the distribution of intrinsic zeros while the stability of intrinsic zeros is still preserved for different values of $\beta$. See also the literature by De la Sen [25], who has similar conclusion by applying different technique.

Next, we display the improvement of the asymptotic properties of discrete system zeros with FROH through an 
TABLE 7: Sampling zeros of the sampled-data system with relative degree two and $\beta=-1 / 2$.

\begin{tabular}{lcc}
\hline$T$ & Approximate values $(47)$ & Exact values \\
\hline \multirow{2}{*}{0.01} & $-0.501195886-0.387172957 i$, & $-0.501218099-0.386984162 i$, \\
& $-0.501195886+0.387172957 i$ & $-0.501218099+0.386984162 i$ \\
0.02 & $-0.502623421-0.386611476 i$, & $-0.502596060-0.386768672 i$, \\
& $-0.502623421+0.386611476 i$ & $-0.502596060+0.386768672 i$ \\
0.05 & $-0.507102272-0.384595337 i$, & $-0.506565882-0.385411371 i$, \\
0.1 & $-0.507102272+0.384595337 i$ & $-0.506565882+0.385411371 i$ \\
0.2 & $-0.516933208-0.375577576 i$, & $-0.512704082-0.381808431 i$, \\
& $-0.516933208+0.375577576 i$ & $-0.512704082+0.381808431 i$ \\
\hline
\end{tabular}

TABLE 8: Sampling zeros of the sampled-data system with relative degree two and $\beta=1$.

\begin{tabular}{lcc}
\hline$T$ & Approximate values (47) & Exact values \\
\hline 0.01 & $0.366015522,-1.370352295$ & $0.366029257,-1.370288147$ \\
0.02 & $0.365962901,-1.374613162$ & $0.365977015,-1.374587969$ \\
0.05 & $0.365635742,-1.386524631$ & $0.365876959,-1.385974102$ \\
0.1 & $0.364115478,-1.403715066$ & $0.364513566,-1.401081359$ \\
0.2 & $0.353215369,-1.422680342$ & $0.369015125,-1.407272983$ \\
\hline
\end{tabular}

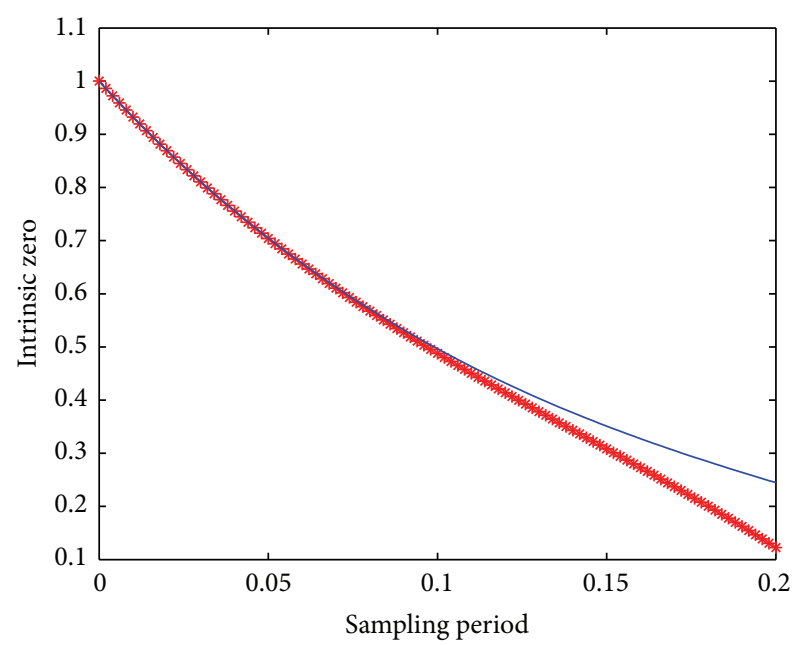

FIGURE 7: Intrinsic zero of sampled-data model with relative degree two.

example of an electronic circuit in the remainder of this section.

Example 3. Consider an electric circuit shown in Figure 10 [15], where $R_{i}(i=1, \ldots, 4)$ and $C_{j}(j=1, \ldots, 3)$ represent resistance and condenser, respectively.

The transfer function with the voltage $e_{i}(t)$ as an input and with the voltage $e_{0}(t)$ as an output is given by

$$
G(s)=\frac{\sum_{k=0}^{1} b_{k} s^{k}}{\sum_{\ell=0}^{3} a_{\ell} s^{\ell}}=K \frac{s+\bar{b}_{0}}{s^{3}+\bar{a}_{2} s^{2}+\bar{a}_{1} s+\bar{a}_{0}},
$$

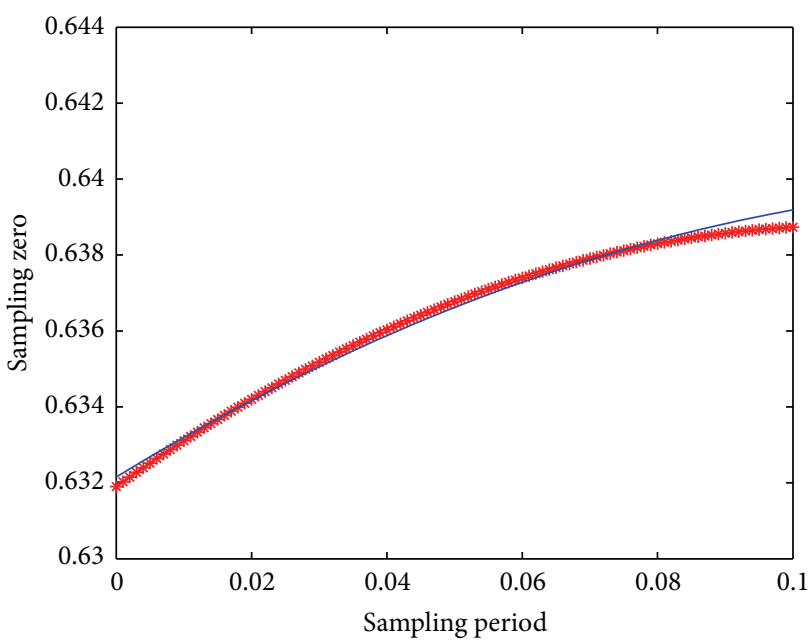

FIGURE 8: Sampling zero of sampled-data model with relative degree two and $\beta=-1 / 2$.

where

$$
K=\frac{b_{1}}{a_{3}}, \quad \bar{b}_{0}=\frac{b_{0}}{b_{1}}, \quad \bar{a}_{0}=\frac{a_{0}}{a_{3}}, \quad \bar{a}_{1}=\frac{a_{1}}{a_{3}}, \quad \bar{a}_{2}=\frac{a_{2}}{a_{3}} .
$$

It is easy to see that the relative degree of transfer function (64) is two. Here, when the parameters are set as $R_{1}=R_{2}=$ $1[\mathrm{k} \Omega], R_{3}=5[\mathrm{k} \Omega], R_{4}=13 / 70[\mathrm{k} \Omega]$, and $C_{1}=C_{2}=$ $C_{3}=1[\mu \mathrm{F}]$, the corresponding discrete-time system with $\mathrm{ZOH}$ has an unstable sampling zero for the sufficiently small sampling periods according to the $\bar{a}_{2}-\bar{b}_{0}<0[21,24]$. In fact, the absolute value of the sampling zero of the discretetime system with $\mathrm{ZOH}$ is 1.006418 for $T=0.001$. The magnitudes of limiting zeros of the corresponding discrete-time system with FROH are shown in Figure 11 for the sampling period $T=0.001$. All the limiting zeros stay inside the unit circle for $-1<\beta<0$ (see also Figure 11). The stability condition can be achieved by means of a suitable choice of the parameter $\beta$ of the improving asymptotic properties.

Remark 14. From Example 3, it has been shown that the limiting zeros of the sampled-data models with FROH can be located inside the stability region by analyzing the improved asymptotic properties while $\mathrm{ZOH}$ fails to do so. In addition, 
TABLE 9: Sampling zeros of the sampled-data system with relative degree two and $\beta=-2$.

\begin{tabular}{lll}
\hline$T$ & Approximate values $(47)$ & Exact values \\
\hline \multirow{2}{*}{0.01} & $-0.497611941+1.938185095 i$, & $-0.497685781+1.938246055 i$, \\
& $-0.497611941-1.938185095 i$ & $-0.497685781-1.938246055 i$ \\
0.02 & $-0.495838288+1.940321092 i$, & $-0.495813965+1.940339611 i$, \\
& $-0.495838288-1.940321092 i$ & $-0.495813965-1.940339611 i$ \\
0.05 & $-0.493521791+1.946037129 i$, & $-0.492000028+1.946432145 i$, \\
0.1 & $-0.493521791-1.946037129 i$ & $-0.492000028-1.946432145 i$ \\
0.2 & $-0.505847953+1.950474754 i$, & $-0.492189267+1.954872572 i$, \\
& $-0.505847953-1.950474754 i$ & $-0.492189267-1.954872572 i$ \\
\hline
\end{tabular}

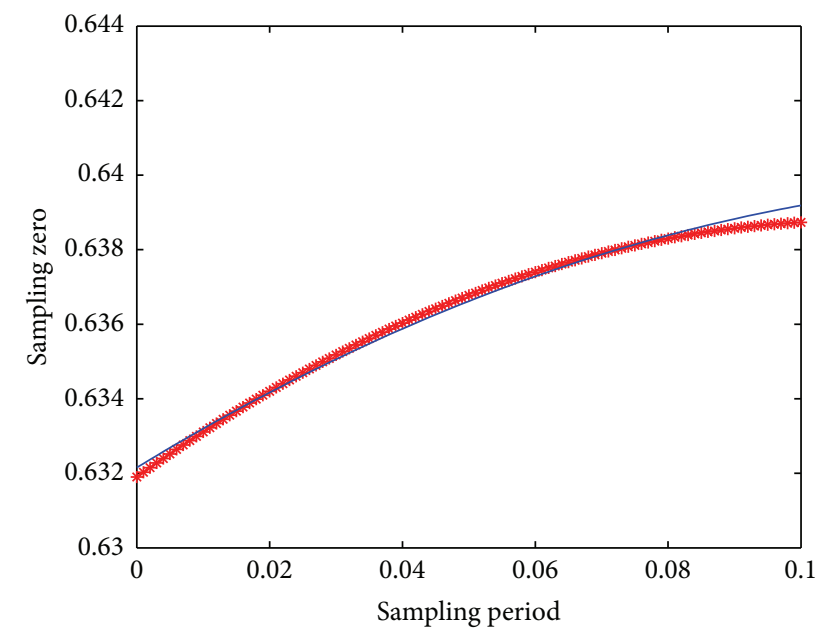

FIGURE 9: Sampling zero of sampled-data model with relative degree two and $\beta=-1 / 2$.

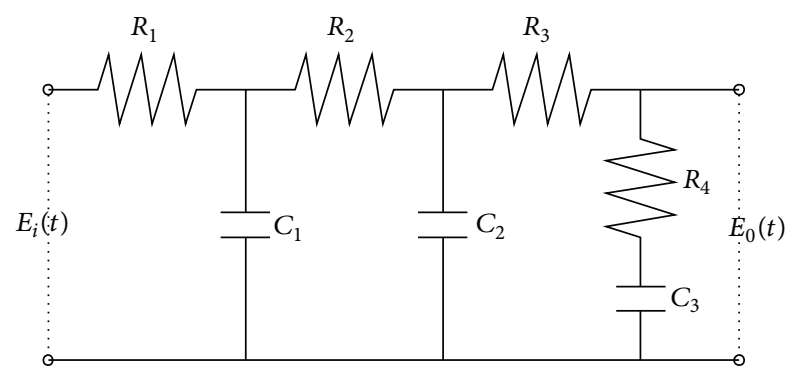

FIgURE 10: The electric circuit plant.

the limiting zeros with AFROH are also stable in some cases due to the same advantages of the FROH and AFROH cases.

\section{Conclusions}

This paper has analyzed the improved asymptotic behavior of limiting zeros for the discrete-time system by using Taylor expansion and the FROH signal reconstruction device. When the normal form representation of continuous-time system with relative degree one or two is discretized, we have given an approximate expression of limiting zeros as power series

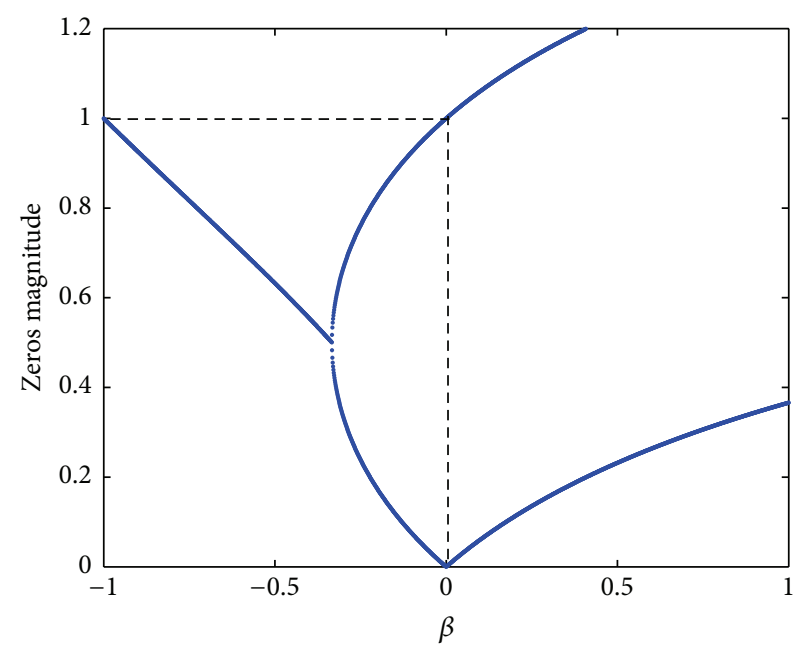

FIGURE 11: The magnitudes of zeros of the sampled-data models with FROH for $T=0.001 \mathrm{~s}$.

expansions with respect to a sampling period up to the thirdorder term. Furthermore, the stability of the sampling zeros is also discussed as the sampling period tends to zero. Finally, it has been shown that $\mathrm{FROH}$ provides advantage over $\mathrm{ZOH}$ with stability of the limiting zeros of sampled-data systems. The idea of this paper is a further extension of the previous results. For a future study, an extension of the approach to multivariable systems is left.

\section{Appendix}

Calculation of (54) and (55). Denote

$$
\begin{gathered}
A_{1}=\left[\begin{array}{lll}
m_{11} & m_{12}+m_{14} & \mathbf{m}_{13}^{T} \\
m_{21} & m_{22}+m_{24} & \mathbf{m}_{23}^{T} \\
\mathbf{m}_{41} & \mathbf{m}_{42}+\mathbf{m}_{44} & M_{43}
\end{array}\right], \\
A_{2}=\left[\begin{array}{lll}
m_{11} & -m_{12} & \mathbf{m}_{13}^{T} \\
m_{21} & -m_{22} & \mathbf{m}_{23}^{T} \\
\mathbf{m}_{41} & -\mathbf{m}_{42} & M_{43}
\end{array}\right] .
\end{gathered}
$$

It is immediate to obtain the value of $\left|A_{1}\right|$ from [21].

Here, consider a matrix $M_{\alpha}$ which is defined by neglecting the higher order terms $O(\cdot)$ with respect to $T$ in the 
matrix $A_{2}$ since the interests lie in the case of small sampling periods $T$.

Multiplying $M_{\alpha}$ by

$$
L_{1}=\left[\begin{array}{cccc}
\frac{1}{T} & \ell_{1} & \mathbf{0}_{n-2}^{T} & \ell_{2} \\
\mathbf{0}_{n}^{T} & I_{n} &
\end{array}\right]^{T},
$$

where

$$
\begin{aligned}
& \ell_{1}=-\frac{1}{m_{0}}\left(\frac{\beta}{2}-\frac{d_{1} \beta}{6} T+\frac{\left(d_{1}^{2}-d_{0}\right) \beta}{24} T^{2}\right), \\
& \ell_{2}=-\frac{\beta T^{2}}{24 m_{0}}, \quad m_{0}=\frac{\beta}{6}-\frac{d_{1} \beta}{24} T+\frac{\left(d_{1}^{2}-d_{0}\right) \beta}{120} T^{2}
\end{aligned}
$$

from the left-hand side leads to

$$
L_{1} M_{\alpha}=\left[\begin{array}{ccc}
\bar{m}_{11} & -\bar{m}_{12} & \overline{\mathbf{m}}_{13}^{T} \\
\bar{m}_{21} & 0 & \overline{\mathbf{m}}_{23}^{T} \\
\overline{\mathbf{m}}_{41} & \mathbf{0}_{n-1} & \bar{M}_{43}
\end{array}\right],
$$

where

$$
\begin{aligned}
\bar{m}_{21}= & -z-2+\frac{3 d_{1}}{4} T+\frac{8 d_{0}-13 d_{1}^{2}}{80} T^{2} \\
& +\frac{28 d_{0} d_{1}-3 d_{1}^{3}-40 \mathbf{c}_{n-3}}{960} T^{3}, \\
\overline{\mathbf{m}}_{23}^{T}= & \frac{\mathbf{c}^{T}}{2} T-\frac{d_{1} \mathbf{c}^{T}}{8} T^{2}+\frac{9 d_{1}^{2} \mathbf{c}^{T}-4 d_{0} \mathbf{c}^{T}-20 \mathbf{c}^{T} P^{2}}{480} T^{3}, \\
\overline{\mathbf{m}}_{41}= & \frac{\mathbf{q}}{4} T^{2}+\frac{8 P \mathbf{q}-5 d_{1} \mathbf{q}^{3}}{48} T^{3}, \\
\bar{M}_{43}= & (1-z) I+P T+\frac{P^{2}}{2} T^{2}+\frac{P^{3}}{6} T^{3} .
\end{aligned}
$$

Noting here that

$$
\begin{aligned}
\delta= & \bar{m}_{21} \times \bar{M}_{43}-\overline{\mathbf{m}}_{23}^{T} \times \overline{\mathbf{m}}_{41} \\
= & {\left[-z-2+\frac{3 d_{1}}{4} T+\frac{8 d_{0}-13 d_{1}^{2}}{80} T^{2}\right.} \\
& +\frac{\left.28 d_{0} d_{1}-3 d_{1}^{3}-40 \mathbf{c}_{n-3} T^{3}\right]}{960} T^{2} T^{3} \\
& \times\left[(1-z) I+P T+\frac{P^{2}}{2} T^{2}+\frac{P^{3}}{6} T^{3}\right]+\frac{\mathbf{q c}^{T}(z-1)}{24} T^{3} \\
= & -z-2+\frac{3 d_{1}}{4} T+\left(-\frac{13 d_{1}^{2}}{80}+\frac{d_{0}}{10}\right) T^{2} \\
& \left.+\left(\frac{7 d_{0} d_{1}}{240}-\frac{3 d_{1}^{3}}{960}-\frac{\mathbf{c}_{n-3}}{12}\right) T^{3}\right] \\
& \times\left[(1-z) I+P T+\frac{P^{2}}{2} T^{2}+\frac{P^{3}}{6} T^{3}\right] .
\end{aligned}
$$

Then,

$$
\left|A_{2}\right|=-\bar{m}_{12} \delta \text {. }
$$

As a result, the calculation is completed.

\section{Acknowledgments}

This work is partially supported by the National Basic Research Program of China ("973” Grant no. 2013CB328903), the National Natural Science Foundation of China (no. 60574003), the Natural Science Foundation Project of CQ CSTC (no. cstc2012jjA40026), and Project no. CDJXS12170006 supported by the Fundamental Research Funds for the Central Universities. The authors also gratefully acknowledge the helpful comments and suggestions of the reviewers, which have improved the presentation of the paper.

\section{References}

[1] K. J. Åström, P. Hagander, and J. Sternby, "Zeros of sampled systems," Automatica, vol. 20, no. 1, pp. 31-38, 1984.

[2] T. Hagiwara and M. Araki, "Properties of limiting zeros of sampled systems," Transactions of the Institute of Electrical Engineers of Japan, vol. 110-C, no. 4, pp. 235-244, 1990 (Japanese).

[3] T. Hagiwara, "Analytic study on the intrinsic zeros of sampleddata systems," IEEE Transactions on Automatic Control, vol. 41, no. 2, pp. 261-263, 1996.

[4] T. Hagiwara, T. Yuasa, and M. Araki, "Stability of the limiting zeros of sampled-data systems with zero- and first-order holds," International Journal of Control, vol. 58, no. 6, pp. 1325-1346, 1993.

[5] S. R. Weller, W. Moran, B. Ninness, and A. D. Pollington, "Sampling zeros and the Euler-Frobenius polynomials," IEEE Transactions on Automatic Control, vol. 46, no. 2, pp. 340-343, 2001.

[6] M. J. Błachuta, "On zeros of pulse transfer functions," IEEE Transactions on Automatic Control, vol. 44, no. 6, pp. C1229C1234, 1999

[7] Y. Hayakawa, S. Hosoe, and M. Ito, "On the limiting zeros of sampled multivariable systems," Systems \& Control Letters, vol. 2, no. 5, pp. 292-300, 1983.

[8] S. R. Weller, "Limiting zeros of decouplable MIMO systems," IEEE Transactions on Automatic Control, vol. 44, no. 1, pp. 129134, 1999.

[9] M. Ishitobi, "A stability condition of zeros of sampled multivariable systems," IEEE Transactions on Automatic Control, vol. 45, no. 2, pp. 295-299, 2000.

[10] S. Liang, M. Ishitobi, W. R. Shi, and X. D. Xian, "On stability of the limiting zeros of discrete-time MIMO systems," Acta Automatica Sinica, vol. 33, no. 4, pp. 439-441, 2007 (Chinese).

[11] K. M. Passino and P. J. Antsaklis, "Inverse stable stampled lowpass systems," International Journal of Control, vol. 47, no. 6, pp. 1905-1913, 1988.

[12] M. Ishitobi, "Stability of zeros of sampled system with fractional order hold," IEE Proceedings, vol. 143, no. 3, pp. 296-300, 1996.

[13] R . Bàrcena, M . de la Sen, and I . Sagastabeitia, "Improving the stability properties of the zeros of sampled systems with fractional order hold," IEE Proceedings, vol. 147, no. 4, pp. 456-464, 2000 . 
[14] R. Bárcena, M. de la Sen, I. Sagastabeitia, and J. M. Collantes, "Discrete control for a computer hard disk by using a fractional order hold device," IEE Proceedings, vol. 148, no. 2, pp. 117-124, 2001.

[15] S. Liang, M. Ishitobi, and Q. Zhu, "Improvement of stability of zeros in discrete-time multivariable systems using fractionalorder hold," International Journal of Control, vol. 76, no. 17, pp. 1699-1711, 2003.

[16] S. Liang and M. Ishitobi, "The stability properties of the zeros of sampled models for time delay systems in fractional order hold case," Dynamics of Continuous, Discrete \& Impulsive Systems B, vol. 11, no. 3, pp. 299-312, 2004.

[17] M. J. Blachuta, "On fast sampling zeros of systems with fractional-order hold," in Proceedings of the American Control Conference, vol. 4, pp. 3229-3230, Arlington, Va, USA, June 2001.

[18] M. Ishitobi, "Properties of zeros of a discrete-time system with fractional order hold," in Proceedings of the 35th IEEE Conference on Decision and Control (CDC '96), pp. 4339-4344, Kobe, Japan, December 1996.

[19] A. Isidori, Nonlinear Control Systems: An Introduction, Springer, Berlin, Germany, 1995.

[20] H. Khalil, Nonlinear Systems, Prentice-Hall, Upper Saddle River, NJ, USA, 2002.

[21] M. Ishitobi, T. Koga, M. Nishi, and S. Kunimatsu, "Asymptotic properties of zeros of sampled-data systems," in Proceedings of the 49th IEEE Conference on Decision and Control (CDC '10), pp. 4952-4957, December 2010.

[22] M. Ishitobi and Q. Zhu, "Zeros of sampled systems with fractional order hold implemented by zero order hold," in Proceedings of the 1997 IEEE International Conference on Intelligent Processing Systems (ICIPS '97), pp. 698-702, Beijing, China, October 1997.

[23] T. Hagiwara, T. Yuasa, and M. Araki, "Limiting properties of the zeros of sampled-data systems with zero- and firstorder holds," in Proceedings of the 31th Conference on Decision and Control (CDC '92), pp. 1949-1954, Tuscon, Ariz, USA, 1992.

[24] S. Liang, M. Ishitobi, T. Koga, and S. Kunimatsu, "On zeros of sampled-data system with relative degree two," in Proceedings of the International Conference on Advanced Mechatronic Systems (ICAMechS '11), pp. 67-71, Zhengzhou, China, August 2011.

[25] M. de la Sen, R. Bárcena, and A. J. Garrido, "On the intrinsic limiting zeros as the sampling period tends to zero," IEEE Transactions on Circuits and Systems I, vol. 48, no. 7, pp. 898900, 2001. 


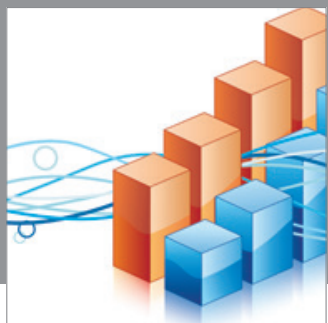

Advances in

Operations Research

mansans

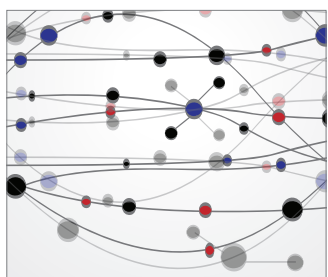

The Scientific World Journal
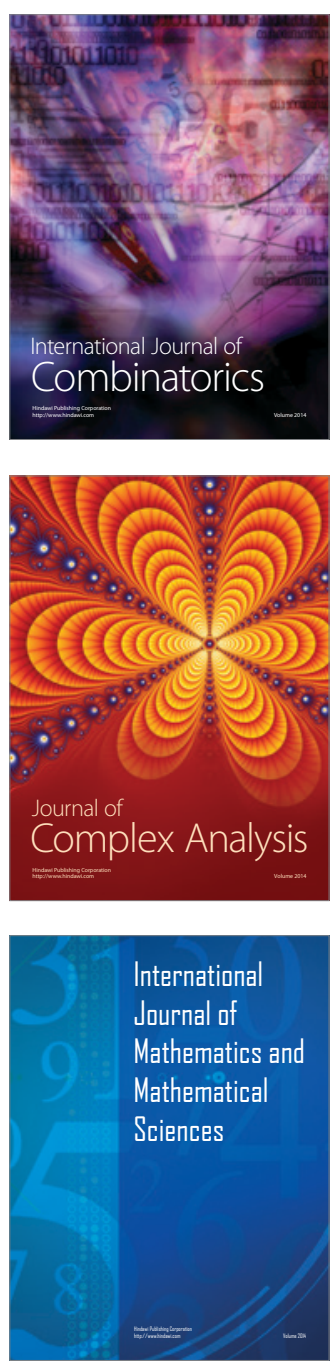
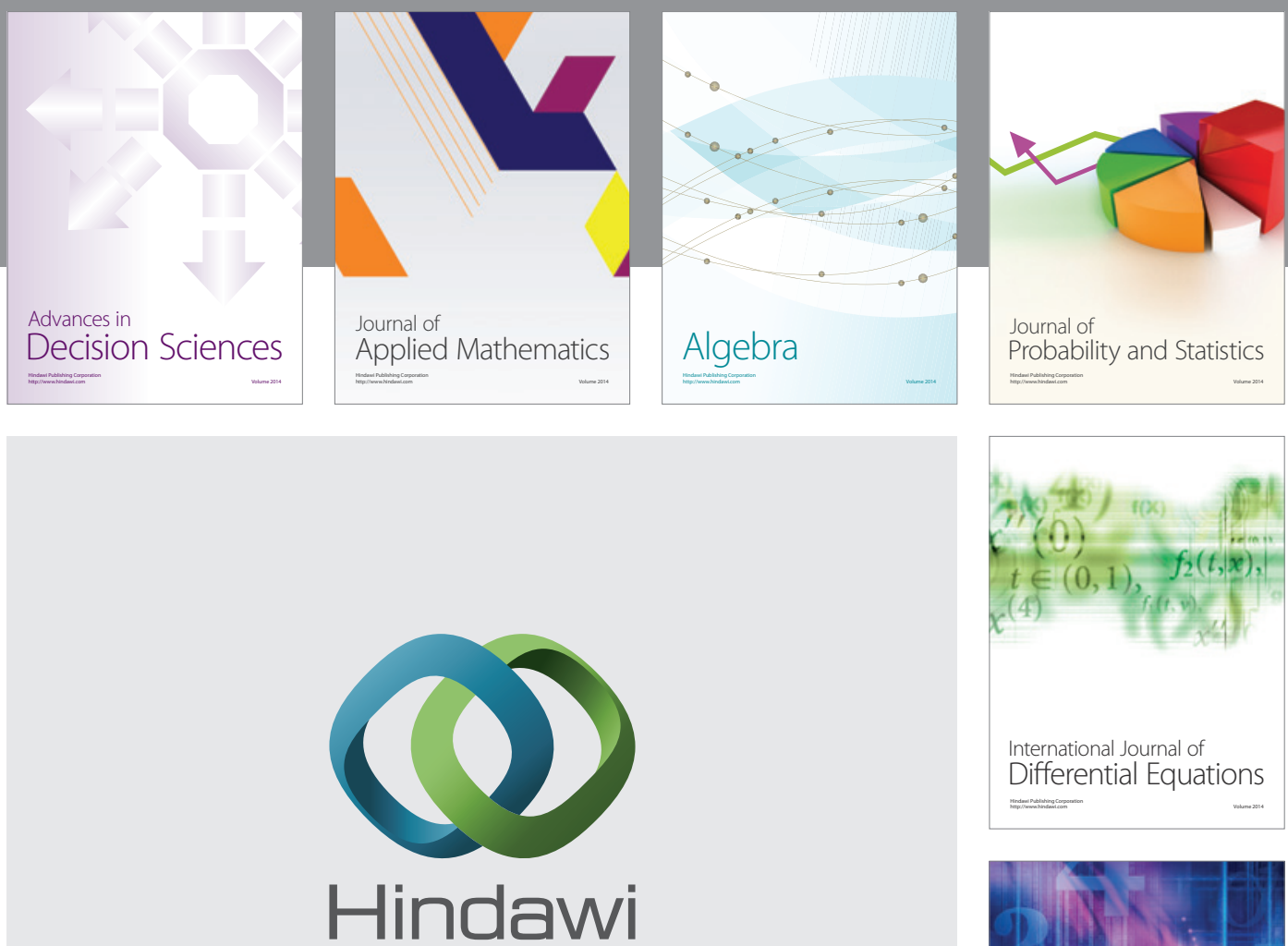

Submit your manuscripts at http://www.hindawi.com
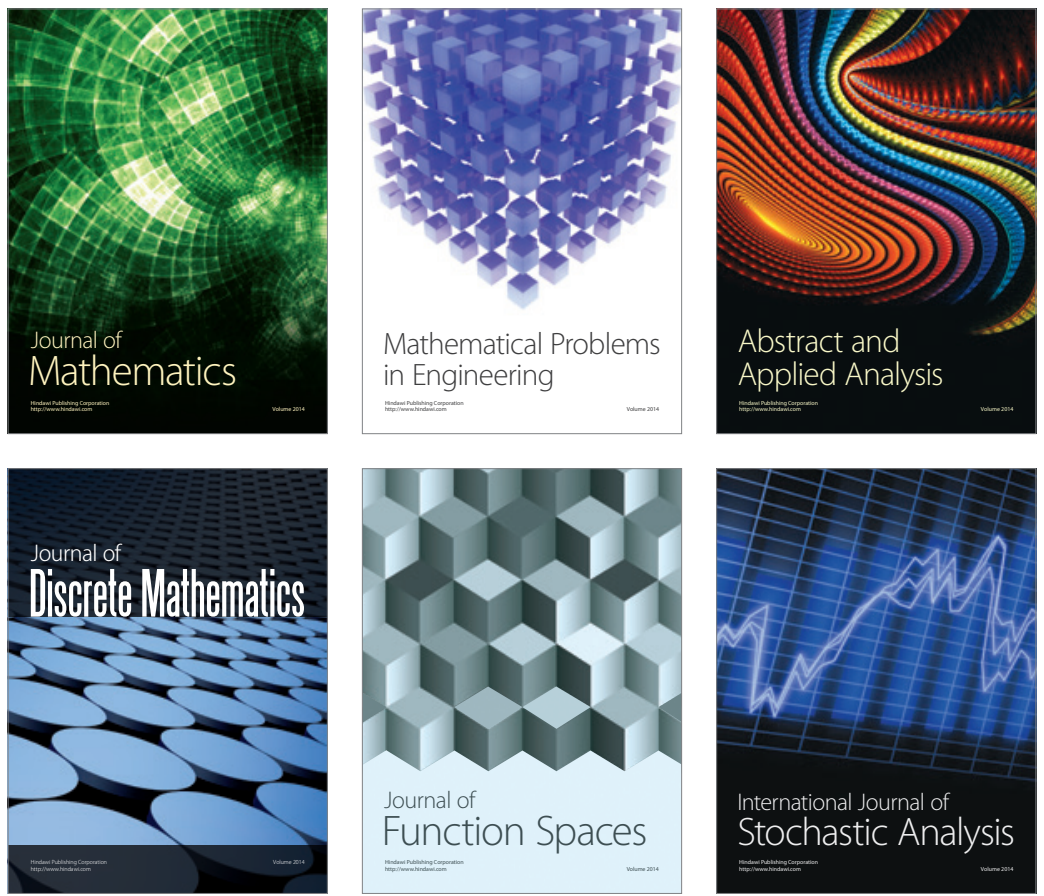

Journal of

Function Spaces

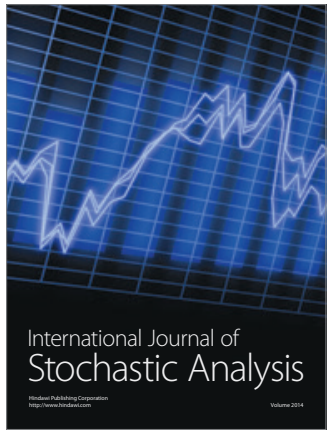

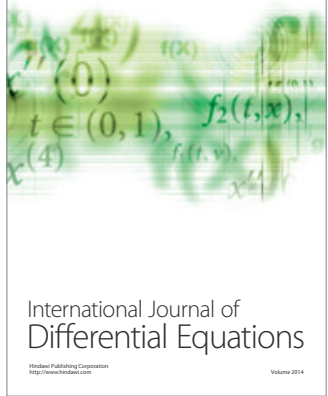
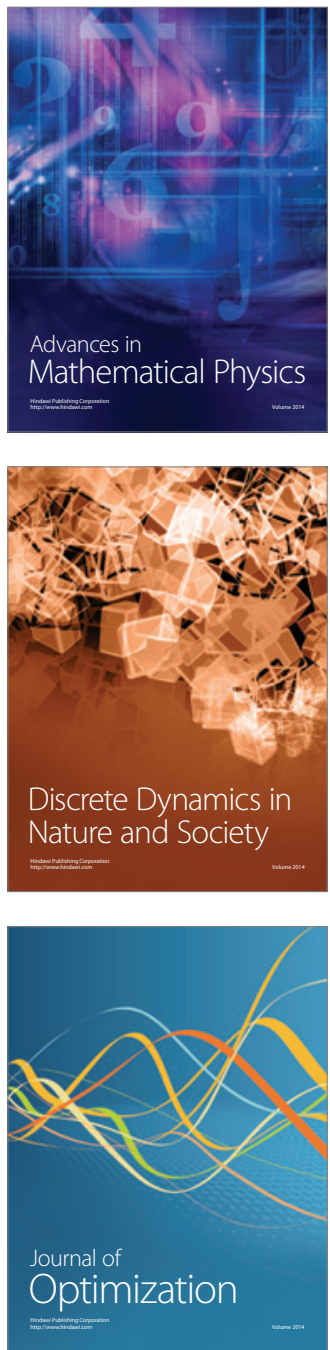